\title{
NLO QCD corrections in Herwig++ with MC@NLO ${ }^{1}$
}

\author{
Stefano Frixione, ${ }^{a, b, 2}$ Fabian Stoeckli, ${ }^{a}$ Paolo Torrielli $^{b}$ and Bryan R. Webber ${ }^{c}$ \\ ${ }^{a}$ PH Department, TH Unit, CERN, \\ CH-1211 Geneva 23, Switzerland \\ ${ }^{b}$ ITPP, EPFL, \\ CH-1015 Lausanne, Switzerland \\ ${ }^{c}$ Cavendish Laboratory, \\ J.J. Thomson Avenue, Cambridge CB3 OHE, U.K. \\ E-mail: Stefano.Frixione@cern.ch, Fabian.Stoeckli@cern.ch, \\ Paolo.Torrielli@epfl.ch, webber@hep.phy.cam.ac.uk
}

ABSTRACT: We present the calculations necessary to obtain next-to-leading order QCD precision with the Herwig++ event generator using the MC@NLO approach, and implement them for all the processes that were previously available from Fortran HERWIG with MC@NLO. We show a range of results comparing the two implementations. With these calculations and recent developments in the automatic generation of NLO matrix elements, it will be possible to obtain NLO precision with Herwig ++ for a much wider range of processes.

KeYwords: QCD Phenomenology

ARXiv EPRINT: 1010.0568

\footnotetext{
${ }^{1}$ Work supported in part by the UK Science and Technology Facilities Council, and by the Swiss National Science Foundation.

${ }^{2}$ On leave of absence from INFN, Sez. di Genova, Italy.
} 


\section{Contents}

1 Introduction $\quad 1$

2 MC@NLO 2

2.1 Generalities 2

2.2 Applications to low-multiplicity processes 4

$\begin{array}{lll}2.3 & \text { MC subtraction terms for Herwig }++ & 6\end{array}$

3 Illustrative results $\quad 8$

3.1 Higgs boson production 8

$\begin{array}{ll}3.2 \text { Top quark pair production } & 10\end{array}$

$\begin{array}{ll}3.3 & \text { Single top production } \\ \end{array}$

$\begin{array}{lll}4 & \text { Conclusions } & 17\end{array}$

$\begin{array}{lr}\text { A Parton showering in Herwig }++ & 17\end{array}$

$\begin{array}{ll}\text { A.1 Final-state emission } & 18\end{array}$

$\begin{array}{ll}\text { A.2 Initial-state emission } & 21\end{array}$

B Construction of MC subtraction terms 22

\section{Introduction}

The high-energy collisions taking place at the Tevatron and at the LHC constitute a severe challenge for any theoretical framework that aims at predicting them in a quantitative manner. On the one hand, the typical final-state multiplicity can range from a few hundred to a few thousand, with an average particle energy of the order of a few GeV. At the same time, the probability is not negligible to have several (up to about ten at the LHC) particles with very large momenta, which can be used as hard probes for studying the highest-energy parton-parton collisions. The task of giving reliable theoretical descriptions of both these aspects of hadron-hadron collisions is a very difficult one, and this is why they are usually not dealt with simultaneously. If one is interested in observables dominated by the kinematics of multi-particle emissions, Parton Shower Monte Carlos (PSMCs) are the method of choice, thanks to their flexibility and to the fact that they are able to give a complete ("exclusive") description of the final states at the level of measurable hadrons. An alternative is given by the so-called resummed computations, which organize the perturbative QCD expansion in terms of the coupling constant $\alpha_{S}$, times the logarithm (possibly to the second power) of a numerically-large ratio of mass scales. The advantage of resummed results over PSMCs is that the former can (at least in principle) be systematically improved by including terms less enhanced by logarithms w.r.t. the dominant ones. However, resummed computations are inclusive, must be performed observable by observable, are very laborious, and rely on a fragmentation-type picture to predict hadron-level observables. 
As far as the predictions of hard-particle cross sections are concerned, they are obtained by a perturbative expansion in $\alpha_{S}$ of the relevant scattering amplitudes. As in the case of resummed computations, this kind of expansion is systematically improvable. However, owing to the complexity of perturbative calculations in QCD, it is now common to have results accurate to NLO (next-to-leading order, i.e. one order more than the one at Born level), while only a handful of results are available to yet higher orders. It has to be stressed that NLO computations give sensible results only in the tails of distributions involving large scales (such as transverse momenta), i.e. for configurations that have a small probability to happen, or for very inclusive observables (such as total rates). On the other hand, it is often the case that interesting physics is characterized by rare events, which justify the importance of higher order computations for the Tevatron and the LHC programmes.

It is clearly desirable to have tools that incorporate the benefits of both PSMCs and perturbative computations, without having their drawbacks. A theoretically-consistent way of matching the two approaches is that defined by the MC@NLO formalism [1]. MC@NLO requires the modification of the parton-level short-distance cross sections used in standard NLO computations, achieved through the insertion of the so-called Monte Carlo (MC) subtraction terms, whose goal is to remove the double counting that would result by naively interfacing an NLO result with a PSMC. The MC subtraction terms can be computed in a process-independent manner, but they are still dependent on the particular PSMC one adopts for the shower phase. In other words, each PSMC requires a set of MC subtraction terms. Although ref. [1] formulated the solution of NLO-PSMC matching in general terms, practical applications there and in subsequent papers have been restricted to the choice of Fortran HERWIG [2-4] as PSMC. Recently, MC subtraction terms relevant to initial-state emissions have been computed for PYTHIA 6.4 [5]. The aim of this paper is to present the computations of the MC subtraction terms for the case of Herwig $++[6,7] .{ }^{1}$ These terms have then been implemented in the MC@NLO computer package. As a consequence, all processes presently implemented in this package can be simulated either with Fortran HERWIG or with Herwig++. We also present here comparisons between the MC@NLO/HERWIG and the MC@NLO/Herwig++ predictions for a few selected observables and processes in hadronic collisions.

This paper is organized as follows. In section 2.1 we summarize the basic features of the MC@NLO formalism, and in section 2.2 we describe their application to the case of lowmultiplicity processes. In section 2.3 we give the general forms of the MC subtraction terms relevant to Herwig++. Section 3 presents the comparisons between results obtained with Herwig++ and Fortran HERWIG in the context of the MC@NLO approach. Our conclusions are reported in section 4. Technical details specific to Herwig ++ are given in appendices A and $\mathrm{B}$.

\section{MC@NLO}

\subsection{Generalities}

The definition of the MC@NLO formalism was given in ref. [1]. We give here a brief summary to establish the notation and terminology. Assuming that the hard process has $2 \rightarrow n$

\footnotetext{
${ }^{1}$ Stand-alone versions of MC@NLO for Herwig++ have been implemented for some processes [8-10].
} 
and $2 \rightarrow n+1$ kinematic configurations at the Born and real-emission level respectively, we associate the real-emission kinematics with the phase-space point $\left(x_{1}, x_{2}, \phi_{n+1}\right)$. A corresponding $2 \rightarrow n$ configuration is obtained from the real-emission kinematics by means of a projection, which is dictated by the structure of the underlying NLO computation. The MC@NLO formalism requires the NLO cross section be computed by means of the so-called FKS subtraction [12]. ${ }^{2}$ The basic idea of the FKS method is the following: for a given $2 \rightarrow n+1$ real-emission process $\mu$, one introduces a set of arbitrary functions $\mathcal{S}_{\mu \mid m}$ (called $\mathcal{S}$ functions) which obey the constraint:

$$
\sum_{m} \mathcal{S}_{\mu \mid m}=1
$$

Each value of the index $m$ is equivalent to the labels of two (strongly-interacting) particles entering the process $\mu$, whose collinear configurations will cause the real-emission matrix element to diverge, and therefore the sum in eq. (2.1) can be interpreted as running over all possible singular regions of process $\mu$. Roughly speaking, the $\mathcal{S}$ functions are constructed in such a way that, in the singular region associated with $m$, all $\mathcal{S}_{\mu \mid m^{\prime}}$ with $m^{\prime} \neq m$ are equal to zero, and $\mathcal{S}_{\mu \mid m}=1 .^{3}$ This leads one to introduce the quantities

$$
\mathcal{M}_{\mu \mid m}^{(n+1)}=\mathcal{S}_{\mu \mid m} \mathcal{M}_{\mu}^{(n+1)}
$$

where $\mathcal{M}_{\mu}^{(n+1)}$ is the real-emission matrix element squared for the process $\mu$. Owing to the properties of the $\mathcal{S}$ functions, each $\mathcal{M}_{\mu \mid m}^{(n+1)}$ has at most one soft and one collinear singularity, which can therefore be subtracted in an essentially trivial manner. In other words, the $\mathcal{S}$ functions effectively achieve a partition of the phase-space, and the index $m$ labels the members of this partition. Using eq. (2.2), one introduces

$$
\frac{d \bar{\Sigma}_{\mu \mid m}^{(n+1)}}{d \phi_{n+1}}=\mathcal{L} \mathcal{M}_{\mu \mid m}^{(n+1)}
$$

$\mathcal{L}$ being the luminosity (i.e. the product of the PDFs), and writes the differential cross section for an observable $O$ at the NLO as follows:

$$
\frac{d \sigma}{d O}=\sum_{\mu, m} \int d x_{1} d x_{2} d \phi_{n+1}\left\{\delta\left(O-O_{n+1}\right) \frac{d \bar{\Sigma}_{\mu \mid m}^{(n+1)}}{d \phi_{n+1}}+\delta\left(O-O_{n}\right) \frac{d \bar{\Sigma}_{\mu \mid m}^{(n)}}{d \phi_{n+1}}\right\}
$$

where $O_{k}$ is the definition of the observable $O$ in terms of $2 \rightarrow k$ kinematic configurations. Eq. (2.4) implicitly defines the $n$-body contribution to the cross section, $\bar{\Sigma}_{\mu \mid m}^{(n)}$, which also enters the MC@NLO generating functional. The notation used here is extremely compact, and in particular one may wonder why an $n$-body contribution is written in terms of the

\footnotetext{
${ }^{2}$ Strictly speaking, MC@NLO may be formulated in terms of any other subtraction formalism, but in practice this has never been done.

${ }^{3}$ The case of soft singularities is technically more involved, but does not present any difficulty of principle. The interested reader is referred to the original publication and to ref. [13] for further details, knowledge of which is irrelevant here.
} 
$n+1$-body phase space. This feature of the subtraction method is explained, together with all the relevant computations, in great detail in refs. [12, 13].

In a completely general case, one can now write the MC@NLO generating functional as follows:

$$
\begin{aligned}
\mathcal{F}_{\mathrm{MC} @ \mathrm{NLO}}=\sum_{\mu, m} \int d x_{1} d x_{2} d \phi_{n+1}\left\{\mathcal{F}_{\mathrm{MC}}^{(n+1)}(\right. & \left.\frac{d \bar{\Sigma}_{\mu \mid m}^{(n+1)}}{d \phi_{n+1}}-\frac{d \bar{\Sigma}_{\mu \mid m}^{(\mathrm{MC})}}{d \phi_{n+1}}\right) \\
& \left.+\mathcal{F}_{\mathrm{MC}}^{(n)}\left(\frac{d \bar{\Sigma}_{\mu \mid m}^{(\mathrm{MC})}}{d \phi_{n+1}}+\frac{d \bar{\Sigma}_{\mu \mid m}^{(n)}}{d \phi_{n+1}}\right)\right\} .
\end{aligned}
$$

We have denoted by $\mathcal{F}_{\text {MC }}^{(k)}$ the generating functional of the Parton Shower Monte Carlo (PSMC) the NLO computation is matched to, where the index $k$ indicates that the initial condition for the shower is given by a $2 \rightarrow k$ partonic configuration. The key point here is the observation that all ingredients necessary for the implementation of the MC@NLO formalism are already present in the computation of the corresponding NLO cross section (2.4), except for the so-called Monte Carlo subtraction terms $\bar{\Sigma}_{\mu \mid m}^{(\mathrm{MC})}$.

As clarified in ref. [1], the MC subtraction terms depend on the PSMC one adopts for showering the hard events. They can be computed by formally expanding the PSMC results to the same order in $\alpha_{S}$ as the corresponding NLO contribution to the parton-level cross section (i.e. that of the real-emission matrix elements). Furthermore, their structures are such that all non trivial process-specific information is contained in the Born matrix elements. These matrix elements are multiplied by kernels whose analytic forms depend solely on the shower variables used by the PSMC to generate the elementary branchings, and on the identities of the partons involved in such branchings. The MC subtraction terms can therefore be computed in a process-independent manner, and subsequently used in eq. (2.5) to simulate a given process whose NLO short-distance cross section has been computed previously.

By construction, the MC subtraction terms cancel locally the divergences of the NLO short-distance cross section. This implies that the quantities that multiply $\mathcal{F}_{\text {MC }}^{(n+1)}$ and $\mathcal{F}_{\text {MC }}^{(n)}$ in eq. (2.5) are separately finite everywhere in the phase space. This allows one to unweight them, and in this way to associate a constant weight with the corresponding kinematic configurations, as is possible with LO-based PSMC; these configurations are called $\mathbb{H}$ and $\mathbb{S}$ events for $(n+1)$-body and $n$-body final states respectively. We point out that a similar unweighting cannot be performed in the context of a pure-NLO computation, eq. (2.4), owing to the fact that the two contributions in the integrand there are associated with different kinematics except on a zero-measure subset of the phase space.

So far the MC subtraction terms have been computed for Fortran HERWIG $[1,11,14]$ and (partly) for PYTHIA [5]. In the following we discuss applications to low-multiplicity processes and computations specific to Herwig ++ .

\subsection{Applications to low-multiplicity processes}

The structure of eqs. (2.5) and (2.4) is suited to the description of processes with arbitrarily large $n$. On the other hand, all practical applications have been restricted to cases for which 
$n=1$ or $n=2$ so far. We stress that this is the number of strongly-interacting particles at the Born level. Accompanying particles such as electroweak bosons or leptons, and decay products of the primary particles, may effectively enlarge $n$, but this does not affect the core of the NLO computation, which is our concern here.

When $n$ is a small, simplifications are possible (but not mandatory) in the structure of eqs. (2.5) and (2.4): for a given $\mu$, one may group together several $\mathcal{S}_{\mu \mid m}$ contributions, owing to the fact that the corresponding kinematic configurations are trivially related to each other. In order to be more precise, we shall give here two specific examples: we consider the two partonic processes

$$
\begin{aligned}
& g\left(p_{1}\right)+g\left(p_{2}\right) \longrightarrow t\left(k_{1}\right)+\bar{t}\left(k_{2}\right)+g\left(k_{3}\right) \\
& u\left(p_{1}\right)+b\left(p_{2}\right) \longrightarrow t\left(k_{1}\right)+d\left(k_{2}\right)+g\left(k_{3}\right)
\end{aligned}
$$

which are part of the real-emission contributions to top-pair and single-top ( $t$ channel) production. We label the initial-state partons with momenta $p_{1}$ and $p_{2}$ by + and respectively, and final-state particles with momenta $k_{i}$ by $f_{i}$. For the two processes in eqs. (2.6) and (2.7) there are four independent $\mathcal{S}$ functions; the corresponding four values of $m$ are:

$$
m=\left(f_{3},+\right), \quad m=\left(f_{3},-\right), \quad m=\left(f_{3}, f_{1}\right), \quad m=\left(f_{3}, f_{2}\right),
$$

which are associated with the configurations in which the final-state gluon $g\left(k_{3}\right)$ may become collinear to one of the initial-state partons $\left(m=\left(f_{3},+\right)\right.$ or $\left.m=\left(f_{3},-\right)\right)$, or to one of the final-state particles $\left(m=\left(f_{3}, f_{1}\right)\right.$ or $\left.m=\left(f_{3}, f_{2}\right)\right)$. At this point, one observes that, in any of these collinear configurations, $p_{1}$ is back-to-back with $p_{2}$, and $k_{1}$ is back-to-back with $k_{2}$. In turn, this implies that when e.g. $g\left(k_{3}\right)$ is collinear with $g\left(p_{1}\right)$, it is anticollinear to $g\left(p_{2}\right)$. In the FKS subtraction formalism, each $\mathcal{S}_{\mu \mid m}$ dictates the choice of the integration variables directly related to the subtraction procedure. In particular, one of these variables is the angle between the two particles identified by the label $m$. It is obvious that, if one given angular variable is suited to performing a subtraction in a collinear region, it will be as well suited to performing it in an anticollinear region. This implies that a single angular variable can be used to deal simultaneously with contributions $m=\left(f_{3},+\right)$ and $m=\left(f_{3},-\right)$, and another one to deal simultaneously with $m=\left(f_{3}, f_{1}\right)$ and $m=\left(f_{3}, f_{2}\right)$.

One further observes that the contributions $m=\left(f_{3}, f_{1}\right)$ and $m=\left(f_{3}, f_{2}\right)$ to eq. (2.6), and the contribution $m=\left(f_{3}, f_{1}\right)$ to eq. (2.7), do not correspond to collinear matrix-element singularities, but only to soft ones. In the FKS procedure, the choice of the angular variable does not play any role in the subtraction of soft singularities, since such a subtraction is dealt with by a variable which is essentially the energy of the parton becoming soft. This implies that, in the case of eq. (2.6), the contributions $m=\left(f_{3}, f_{1}\right)$ and $m=\left(f_{3}, f_{2}\right)$ can be treated together with those $m=\left(f_{3}, \pm\right)$. In the case of eq. (2.7), on the other hand, the contribution $m=\left(f_{3}, f_{1}\right)$ can be treated simultaneously with either $m=\left(f_{3}, \pm\right)$, or with $m=\left(f_{3}, f_{2}\right)$. Although either choice is possible, in ref. [14] the latter, more logical, option was adopted.

The bottom line is that, owing to the simplified kinematics of $2 \rightarrow 1$ or $2 \rightarrow 2$ processes, some of the contributions in the sum over $m$ in eqs. (2.5) and (2.4) can be dealt with 
together. In such cases, it is still convenient to use the notation introduced in eqs. (2.5) and (2.4), but $m$ will need be understood as a set of pairs of particle labels, rather than as a single pair. For the specific examples considered above, we shall have

$$
\begin{aligned}
& m=\left\{\left(f_{3},+\right),\left(f_{3},-\right),\left(f_{3}, f_{1}\right),\left(f_{3}, f_{2}\right)\right\}, \\
& m=\left\{\left(f_{3},+\right),\left(f_{3},-\right)\right\}, \quad m=\left\{\left(f_{3}, f_{1}\right),\left(f_{3}, f_{2}\right)\right\},
\end{aligned}
$$

for the processes in eqs. (2.6) and (2.7) respectively. Therefore, in the case of top-pair production, the sums over $m$ in eqs. (2.5) and (2.4) contain only one term and, thanks to eq. (2.1), it is actually not necessary to introduce the $\mathcal{S}$ function in order to perform the computation. The case of single-top is only slightly more complicated. The sums over $m$ contain two contributions; in the original publication, ref. [14], the two values of $m$ in eq. (2.10) have been denoted by IN and OUT respectively.

We conclude this section by pointing out that, regardless of the number of final-state particles $n$, contributions $m=\left(f_{i},+\right)$ and $m=\left(f_{i},-\right)$ for a given particle label $f_{i}$ can be always treated simultaneously in the context of the FKS subtraction - see appendix E of ref. [13] for a discussion on this matter.

\subsection{MC subtraction terms for Herwig ++}

As discussed previously, the MC subtraction terms $\bar{\Sigma}_{\mu \mid m}^{(\mathrm{MC})}$ are determined by formally expanding the results of the PSMC in $\alpha_{S}$, and by keeping the contribution that has the same power in $\alpha_{S}$ as the NLO cross section (which we shall denote by $\mathcal{O}\left(\alpha_{S}^{b+1}\right)$ ). This contribution can be written in full generality as follows:

$$
\left.d \sigma\right|_{\mathrm{MC}}=\left.\sum_{\mu, m} \sum_{L \in m} \sum_{l} d \sigma_{\mu}^{(L, l)}\right|_{\mathrm{MC}} .
$$

The sum over $\mu$ has precisely the same meaning (and range) as that in eqs. (2.5) and (2.4), since the order in $\alpha_{S}$ at which we are working is the same as in those equations, and hence the partonic processes that contribute to the cross sections are the same. The sums over $m$ and $L$ can be understood as follows. At $\mathcal{O}\left(\alpha_{S}^{b+1}\right)$, the PSMC gets contributions from the diagrams that can be constructed by attaching to Born-level diagrams all possible $1 \rightarrow 2$ (QCD) branchings stemming from external legs. These diagrams are therefore a subset of those contributing to real-emission matrix elements, and in particular are all diagrams that may give rise to collinear and/or soft singularities. Hence, they can be identified by means of the index $m$ introduced previously. According to the discussion in section 2.2, each $m$ is a set of pairs of particle labels, with one (in the straightforward implementation described in section 2.1) or more (in the case simplifications are possible) elements. Either way, the index $L$ in eq. (2.11) runs over the elements of a given $m$. This implies that each element in the double sum over $m$ and $L$ in eq. (2.11) identifies unambiguously one "branching" in a real-emission configuration. Note, therefore, that at fixed $\mu$ there is a one-to-one correspondence between $L$ and a particle in the underlying Born-level process; such a particle may thus be referred to as the branching leg. Finally, for each branching leg the PSMC may give rise to different showers, depending on the colour partner of the 
branching leg. The sum over the choice of colour partners is that over the index $l$ in eq. (2.11); note that, at fixed $L$, a given particle can play the role of colour partner more than once, depending on the colour flows of the underlying Born process.

The similarities between Fortran HERWIG (denoted henceforth by HERWIG6) and Herwig++ are such that the forms of the cross sections $\left.d \sigma_{\mu}^{(L, l)}\right|_{\mathrm{MC}}$ are the same as those given in ref. [14]. They read:

$$
\begin{aligned}
& \left.d \sigma_{\mu}^{(+, l)}\right|_{\mathrm{MC}}=\left.\frac{1}{z_{+}} f_{a}^{\left(H_{1}\right)}\left(\bar{x}_{1 i} / z_{+}\right) f_{b}^{\left(H_{2}\right)}\left(\bar{x}_{2 i}\right) d \hat{\sigma}_{\mu}^{(+, l)}\right|_{\mathrm{MC}} d \bar{x}_{1 i} d \bar{x}_{2 i}, \\
& \left.d \sigma_{\mu}^{(-, l)}\right|_{\mathrm{MC}}=\left.\frac{1}{z_{-}} f_{a}^{\left(H_{1}\right)}\left(\bar{x}_{1 i}\right) f_{b}^{\left(H_{2}\right)}\left(\bar{x}_{2 i} / z_{-}\right) d \hat{\sigma}_{\mu}^{(-, l)}\right|_{\mathrm{MC}} d \bar{x}_{1 i} d \bar{x}_{2 i}, \\
& \left.d \sigma_{\mu}^{\left(f_{\alpha}, l\right)}\right|_{\mathrm{MC}}=\left.f_{a}^{\left(H_{1}\right)}\left(\bar{x}_{1 f}\right) f_{b}^{\left(H_{2}\right)}\left(\bar{x}_{2 f}\right) d \hat{\sigma}_{\mu}^{\left(f_{\alpha}, l\right)}\right|_{\mathrm{MC}} d \bar{x}_{1 f} d \bar{x}_{2 f},
\end{aligned}
$$

where, consistently with section 2.2 , we have denoted by + and - the two initial-state branching legs, and with $f_{\alpha}$ the final state ones. In eqs. (2.12)-(2.14) we have shortened the notation, denoting e.g. $L=\left(f_{i}, \pm\right)$ and $L=\left(f_{i}, f_{\alpha}\right)$ simply by \pm and $f_{\alpha}$ respectively, for any given particle label $f_{i}$. This does not result in any ambiguities, since clearly the equations above are formally identical for any $f_{i}$.

The distribution function of parton $p$ in hadron $H_{i}$ is denoted by $f_{p}^{\left(H_{i}\right)}$, and the identities of initial-state partons $a$ and $b$ depend on $\mu$. In spite of the fact that eqs. (2.12) and (2.13) are formally identical to the analogous equations of ref. [14], we stress that the variables $z_{ \pm}$are different in the two cases, being (one of) the shower variables of Herwig ++ here, and of HERWIG6 in ref. [14]. On the other hand, the variables $\bar{x}_{1 i}, \bar{x}_{2 i}, \bar{x}_{1 f}$, and $\bar{x}_{2 f}$ (i.e. the Bjorken $x$ 's used by the PSMC) are the same for Herwig++ as for HERWIG6. This statement is trivial in the context of standalone PSMC usage (given that the Bjorken $x$ 's are just integration variables), but it is not when eqs. (2.12)-(2.14) are used for the construction of the MC subtraction terms. In such a case, in fact, the PSMC Bjorken $x$ 's need be computed in terms of those used in the NLO computation, and this is done in MC@NLO by means of the procedure called event projection, described in details in ref. [1]. Ultimately, event projection depends on the treatment of the kinematics of the hard process by the PSMC. Since it is possible to use the same procedure in Herwig++ as in HERWIG6, it follows that the variables above have the same analytic forms in the two cases.

The short-distance cross sections that appear in eqs. (2.12)-(2.14) read as follows:

$$
\begin{aligned}
\left.d \hat{\sigma}_{\mu}^{( \pm, l)}\right|_{\mathrm{MC}} & =\frac{\alpha_{S}}{2 \pi} \frac{d \tilde{q}_{ \pm}^{2}}{\tilde{q}_{ \pm}^{2}} d z_{ \pm} P_{a^{\prime} b^{\prime}}\left(z_{ \pm}\right) d \bar{\sigma}_{\mu^{\prime}}^{( \pm, l)} \Theta_{\text {dead }}^{( \pm, l)} \\
\left.d \hat{\sigma}_{\mu}^{\left(f_{\alpha}, l\right)}\right|_{\mathrm{MC}} & =\frac{\alpha_{S}}{2 \pi} \frac{d \tilde{q}_{f_{\alpha}}^{2}}{\tilde{q}_{f_{\alpha}}^{2}} d z_{f_{\alpha}} P_{a^{\prime} b^{\prime}}\left(z_{f_{\alpha}}, \tilde{q}_{f_{\alpha}}^{2}\right) d \bar{\sigma}_{\mu^{\prime}}^{\left(f_{\alpha}, l\right)} \Theta_{\text {dead }}^{\left(f_{\alpha}, l\right)}
\end{aligned}
$$

Their forms follow in a straightforward manner from considering how any (collinear-based) PSMC deals with $1 \rightarrow 2$ branchings. They are constructed by multiplying the Born-level cross section $d \bar{\sigma}_{\mu^{\prime}}^{(L, l)}$ by the relevant collinear or quasi-collinear Altarelli-Parisi splitting kernels (here both denoted by $P$ ), times the measure that appears in the Sudakov form factors. This is PSMC-specific, and depends in particular on the shower variables. Those 
of Herwig ++ are denoted by $z_{L}$ and $\tilde{q}_{L}^{2}$, and are discussed in appendix A. Finally, the $\Theta$ functions in eqs. (2.15) and (2.16) are due to the fact that in general there are phase-space regions where the PSMC cannot emit radiation (called dead zones). The dead zones are PSMC-specific; those relevant to Herwig ++ are given in appendix A. The identities of the partons involved in the branching, $a^{\prime}$ and $b^{\prime}$, and of the Born process, $\mu^{\prime}$, are fully determined by $\mu$ and by $(L, l)$ - their particular values are irrelevant in what follows.

\section{Illustrative results}

In this section we present some illustrative results of the implementation of MC@NLO for Herwig++, compared in each case with results from the HERWIG6 implementation. Both are available for the same range of processes: Higgs boson, single vector boson, vector boson pair, heavy quark pair, single top (with and without associated $W$ ), lepton pair and associated Higgs $+W / Z$ production in hadron collisions. In most processes the results are very similar. This is reassuring, since the showering algorithms in the two event generators, and the corresponding modified subtractions in the NLO calculations, are quite different. Where differences are seen, they can be ascribed to changes in the parton showering algorithm, particularly for heavy quarks, and to different modelling of non-perturbative physics. There is an overall tendency for slightly more but softer gluon radiation in Herwig++. Some of our results are presented at the parton level, and some other at the hadron level; our jets are defined by clustering stable hadrons. We have verified that parton-level results are fairly close to hadron-level ones for both HERWIG6 and Herwig++, and for this reason we refrain from presenting the comparisons between the predictions of the two event generators in both cases. One notable exception will be discussed in section 3.3.

For these comparisons MC@NLO was interfaced to the current versions of the event generators, Herwig++ v2.4.2 and HERWIG v6.520. All results are for the LHC at centreof-mass energy $7 \mathrm{TeV}$, using the CTEQ6.6 NLO parton distributions [16], and without the inclusion of the underlying event. Finally, we have switched the intrinsic $p_{T}$ off in both Herwig ++ and HERWIG6.

\subsection{Higgs boson production}

In general the MC@NLO results on electroweak boson production using Herwig++ and HERWIG6 show few significant differences. Changes in the boson transverse momentum distributions at low $p_{T}$ can be ascribed to the softer QCD radiation in Herwig++. For example, figure 1 shows the transverse momentum and rapidity distributions of a StandardModel Higgs boson with a mass of $160 \mathrm{GeV}$. Compared to HERWIG6, an increase is observed for $p_{T}<20 \mathrm{GeV}$, due to the slight softening of QCD radiation, but the rapidity distribution is not affected.

Figure 2 shows the charged lepton correlations when the Higgs boson decays to $W W \rightarrow$ $l \nu l \nu$. These distributions are not much affected by soft QCD radiation and there is close agreement between the two implementations. 

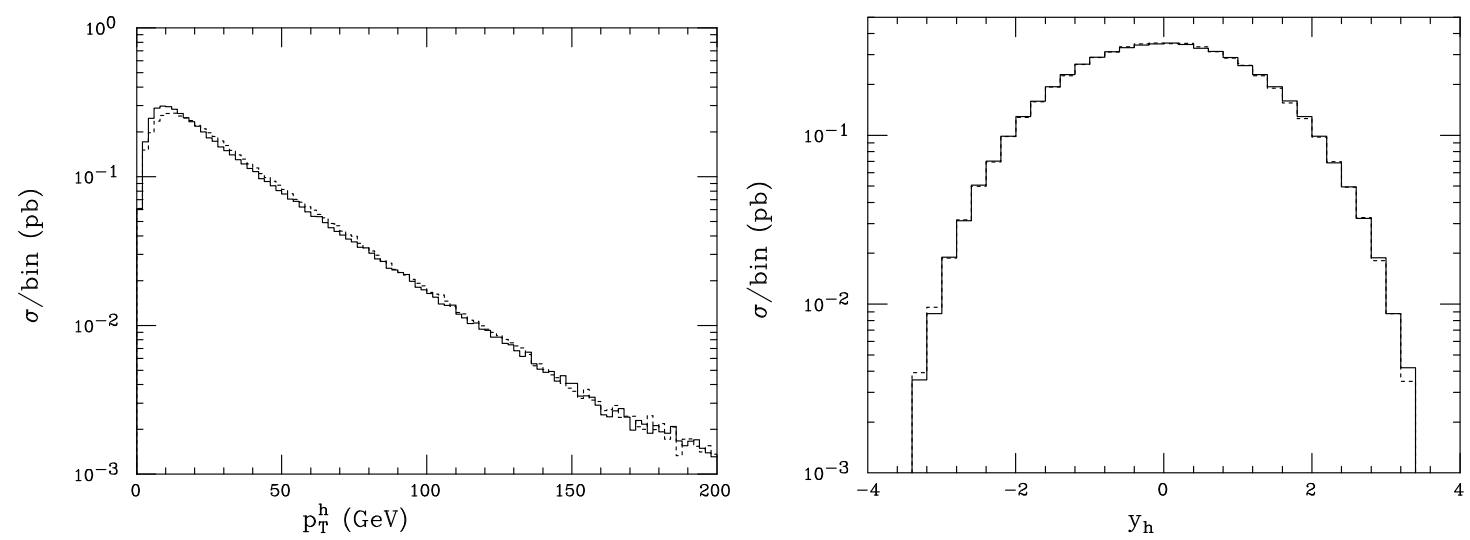

Figure 1. MC@NLO results on Higgs boson production: Higgs transverse momentum (left) and rapidity (right) distributions with Herwig++ (solid) and HERWIG6 (dashed).
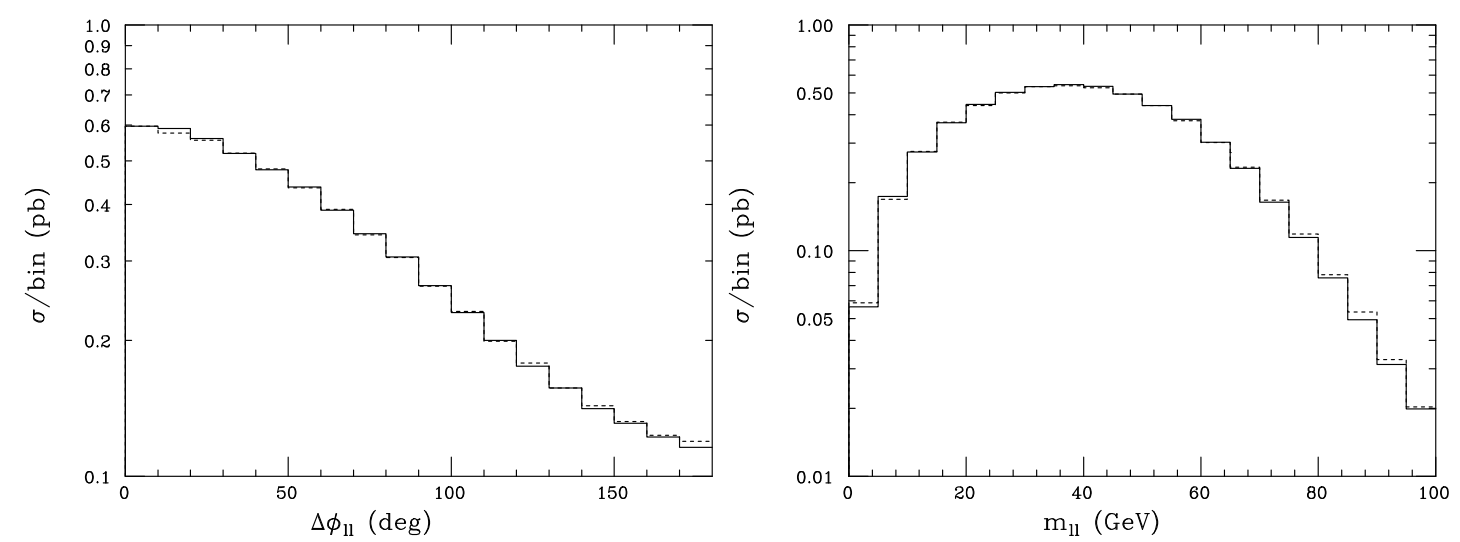

Figure 2. MC@NLO results on Higgs $\rightarrow W W \rightarrow l \nu l \nu$ for Herwig++ (solid) and for HERWIG6 (dashed). Spin correlations are included. Left panel: azimuthal angle between the charged leptons. Right panel: dilepton invariant mass.

In refs. $[17,18]$ the effects of acceptance cuts on searches for a Higgs boson in the $W W \rightarrow l \nu l \nu$ decay channel were studied. The cuts were chosen to mimic realistic event selection as applied in experimental searches for Higgs bosons in this channel:

1. the leading charged lepton should be within $|\eta|<2$ and $30 \mathrm{GeV}<p_{T}<55 \mathrm{GeV}$;

2. the trailing charged lepton has to fulfill $|\eta|<2$ and $p_{T}>25 \mathrm{GeV}$;

3. the invariant mass of the charged lepton pair is restricted to $12 \mathrm{GeV}<m_{l l}<40 \mathrm{GeV}$;

4. the missing transverse energy has to exceed $50 \mathrm{GeV}$;

5. the opening angle between the two charged leptons in the transverse plane has to be smaller than $\phi_{l l}<\pi / 4$;

6. there should be no jet with $p_{T}>25 \mathrm{GeV}$ and $|\eta|<2.5$ in the event (jet-veto). 


\begin{tabular}{|c|c|c|c|}
\hline Generator & $\sigma_{\text {inc }}[\mathrm{fb}]$ & $\sigma_{\text {acc }}[\mathrm{fb}]$ & $\varepsilon[\%]$ \\
\hline Herwig++ & 5.76 & 0.479 & $8.32 \pm 0.04$ \\
HERWIG6 & 5.76 & 0.444 & $7.71 \pm 0.04$ \\
\hline
\end{tabular}

Table 1. MC@NLO results on the acceptance for Higgs $\rightarrow W W \rightarrow l \nu l \nu$ after the cuts indicated in the text. The errors on the efficiencies are the statistical uncertainties.

Higher-order QCD corrections were seen to have significant impact on the efficiency of these cuts, and MC@NLO with HERWIG6 was found to give good agreement with NNLO calculations of acceptance effects. In table 1 we show the corresponding results for MC@NLO with Herwig++. As can be seen, the efficiencies predicted by the two event generators are similar, though not identical. The cuts applied can be divided into two sets: cuts on kinematic variables of the final state leptons (such as invariant mass, transverse momentum, rapidity), and cuts on the hadronic structure of the event (jet-veto). While the acceptances of the leptonic cuts are very similar between Herwig++ and HERWIG6 (see also figure 2), the difference in the overall efficiency arises from the different hadronic structure in the low momentum region (see also figure 1). This demonstrates again how the uncertainty on a jet-veto efficiency has to be studied very carefully when applied in experimental searches.

\subsection{Top quark pair production}

The HERWIG6 and Herwig++ event generators differ significantly in their treatment of heavy quarks. The main differences are

1. The kinematics of the parton shower. The energy-angle shower variables used in HERWIG6 led to a sharp angular cutoff at $\theta \sim m / E$ in the radiation pattern from a quark of mass $m$ and energy $E$ (the so-called dead cone), whereas Herwig++ uses a more covariant formulation that allows emission at lower angles [19].

2. The introduction of mass corrections to the parton splitting functions in Herwig ++ , following the 'quasi-collinear' prescription of ref. [20].

3. An improved treatment of QCD radiation in top decay in Herwig++, developed in refs. [19, 21], which ensures a better angular distribution and removes the need for an ad hoc infrared cutoff.

As a result of these improvements, differences in the results of MC@NLO with HERWIG6 and Herwig ++ for processes involving heavy quarks are somewhat more pronounced than those in electroweak boson production. The most significant effect is a softer spectrum of QCD radiation in top quark production and decay in Herwig++.

Figures 3 and 4 show the resulting effects on the $t \bar{t}$ transverse momentum spectrum and azimuthal separation, respectively. In these and subsequent figures we also show the distributions after acceptance cuts. For top quark pair production, these are defined as follows: in figures 3 and 4 , we require the transverse momenta of the $t$ and $\bar{t}$ to be larger than $30 \mathrm{GeV}$, and the absolute values of their rapidities to be smaller than 2.5. On the other 

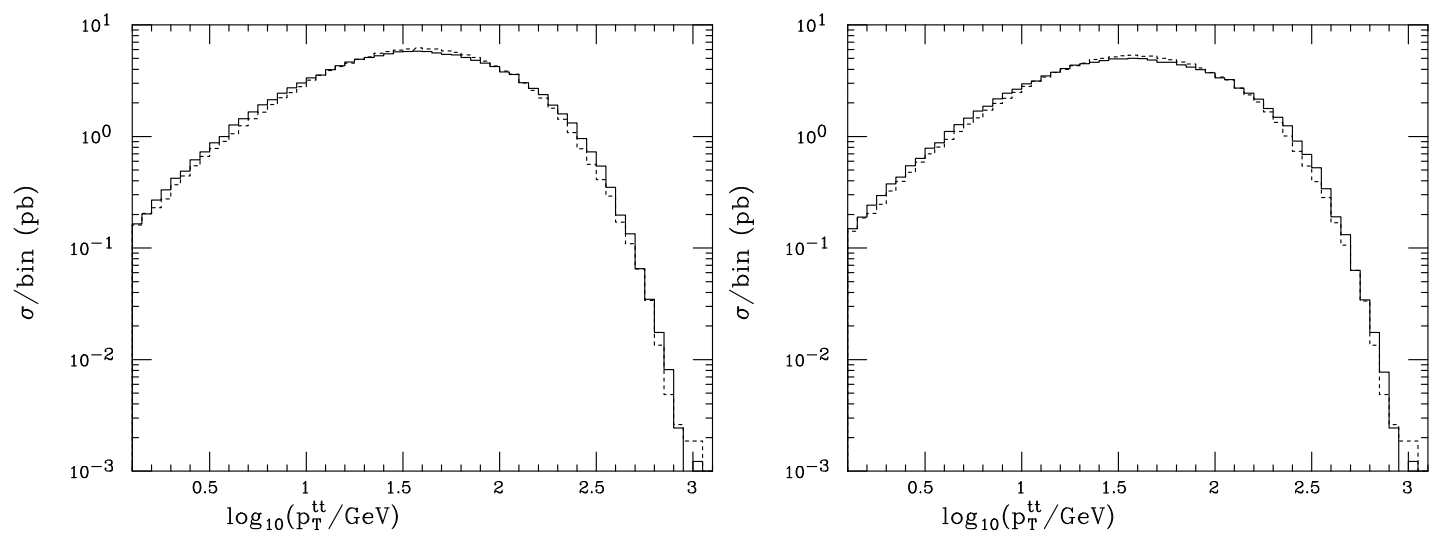

Figure 3. MC@NLO results on top quark pair production: $\log _{10}$ of the $t \bar{t}$ transverse momentum distribution with Herwig++ (solid) and HERWIG6 (dashed). Left/right panel: without/with acceptance cuts.
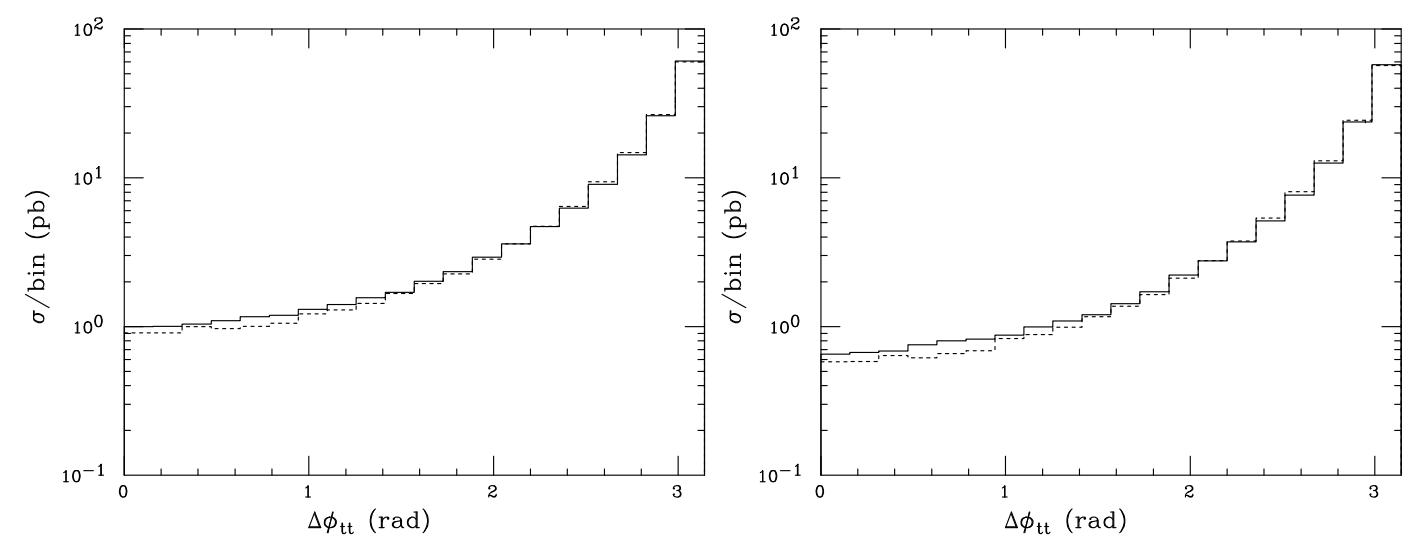

Figure 4. MC@NLO results on top quark pair production: $t \bar{t}$ azimuthal separation with Herwig++ (solid) and HERWIG6 (dashed). Left/right panel: without/with acceptance cuts.

hand, in figures $5-8$, which were obtained by letting the top quarks decay leptonically, we require the transverse momenta (absolute values of the rapidities) of the "visible" decay products, i.e. the $b$ and $\bar{b}$ quarks and the charged leptons $l^{ \pm}$, to be larger than $30 \mathrm{GeV}$ (smaller than 2.5).

Figures 5 and 6 show aspects of the distribution of $b$ quarks from decay of the top pair: the transverse momentum relative to the beam direction in figure 5 and relative to the direction of motion of the parent top in figure 6. Decay angular correlations due to the top polarization are included following the prescription of ref. [15]. Here again there is some softening and smearing of the distribution in Herwig++. A corresponding softening of the transverse momentum and invariant mass distributions of the pair of $b$ quarks may be seen in figures 7 and 8 . 

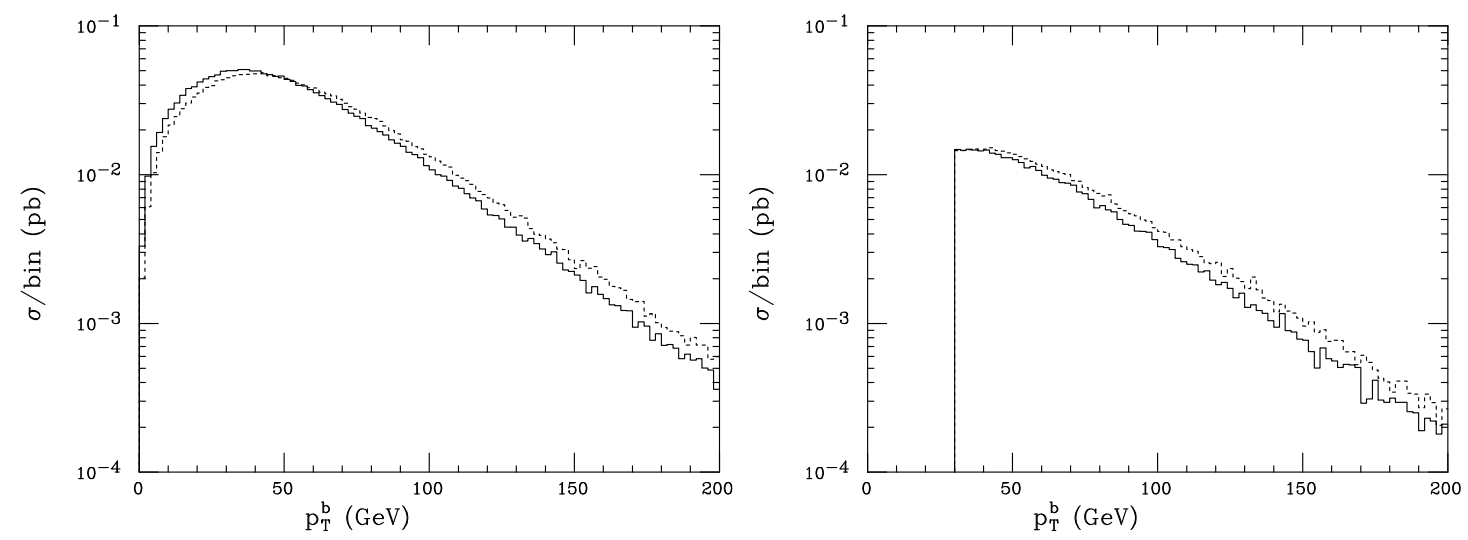

Figure 5. MC@NLO results on top quark pair production: $b$-quark transverse momentum distribution with Herwig ++ (solid) and HERWIG6 (dashed). Left/right panel: without/with acceptance cuts.
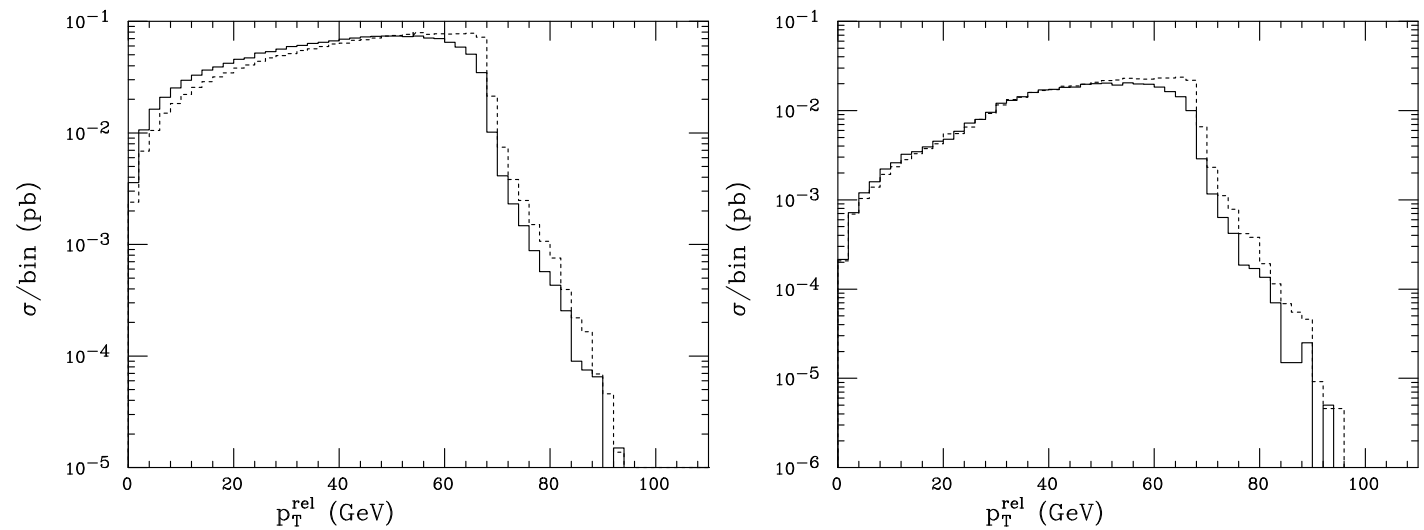

Figure 6. MC@NLO results on top quark pair production: $b$-quark transverse momentum (relative to the direction of flight of the top) distribution with Herwig ++ (solid) and HERWIG6 (dashed). Left/right panel: without/with acceptance cuts.

\subsection{Single top production}

Comparisons between results on single top production ${ }^{4}$ from MC@NLO with Herwig++ and HERWIG6 reveal similar basic features to those in top quark pair production. There is a general softening of distributions due to softer QCD radiation in Herwig++, illustrated here by the top plus leading jet transverse momentum distribution, figure 9 . The jets have been defined with the $k_{T}$ algorithm as implemented by FastJet [22, 23], with $R=0.5$ and by requiring each jet to have transverse momentum larger than $10 \mathrm{GeV}$. Furthermore, we have eliminated from the list of our jets the one that contains the $b$-flavoured hadron that emerges from the decay of the top. Apart from the jet cuts, when imposing acceptance

\footnotetext{
${ }^{4}$ We have limited ourselves to considering here top quark production (i.e., antitop quark production is not included) in the $t$ channel.
} 

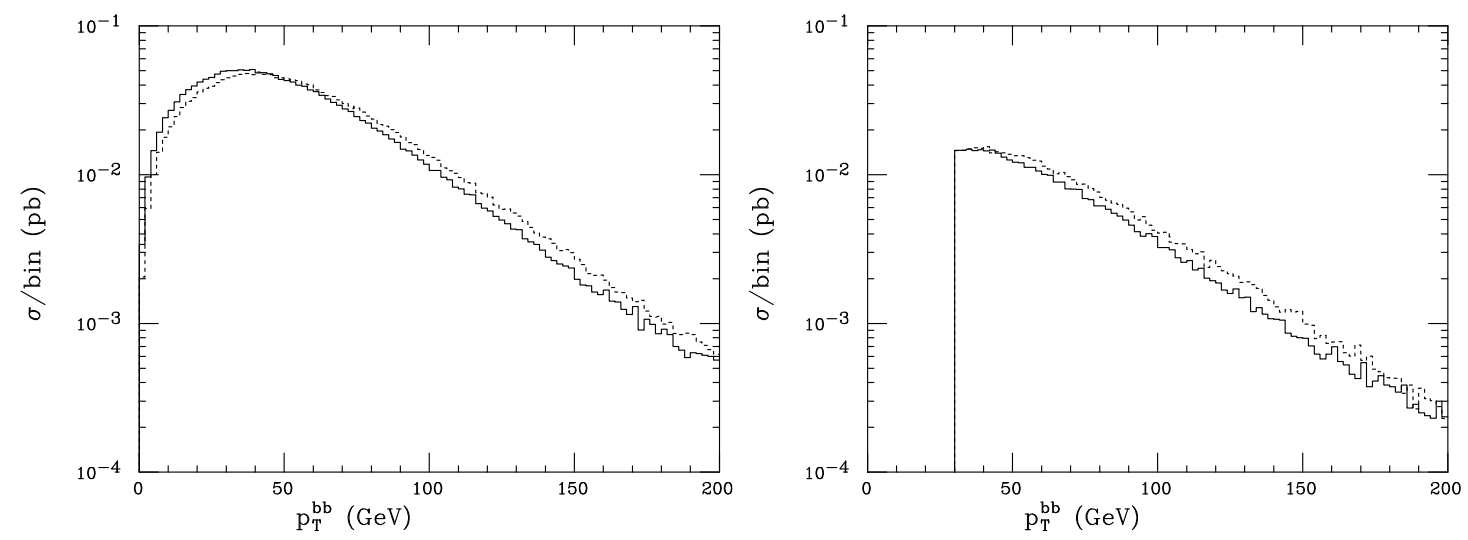

Figure 7. MC@NLO results on top quark pair production: $b \bar{b}$ transverse momentum distribution with Herwig ++ (solid) and HERWIG6 (dashed). Left/right panel: without/with acceptance cuts.
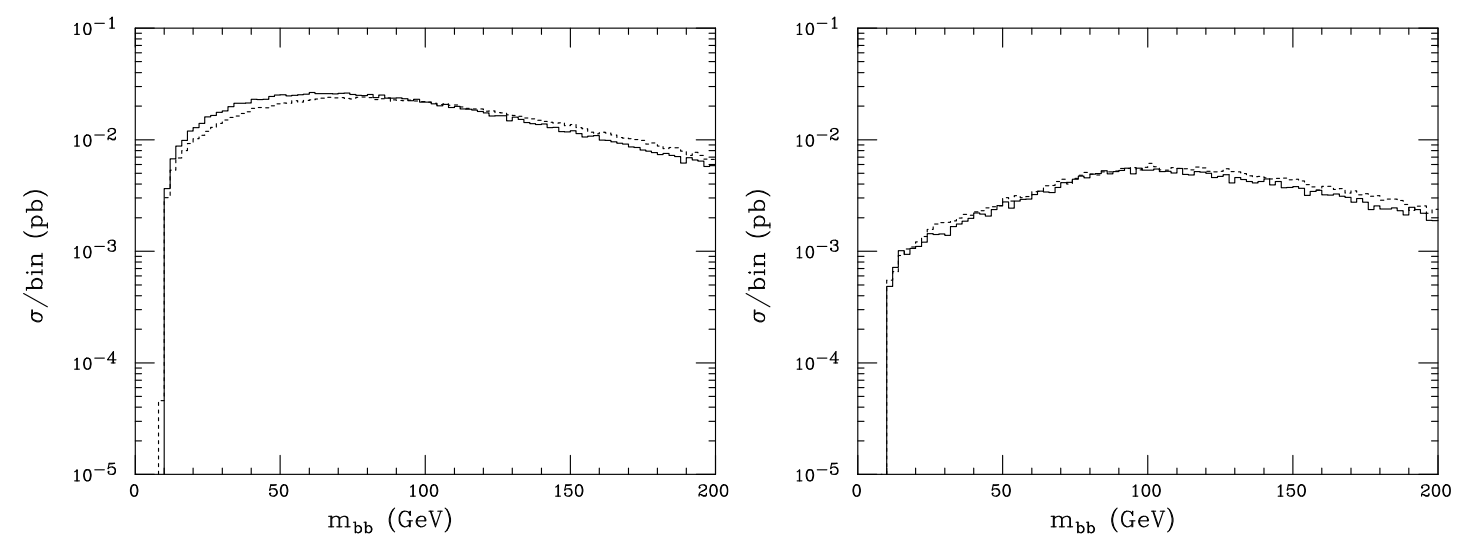

Figure 8. MC@NLO results on top quark pair production: $b \bar{b}$ invariant mass distribution with Herwig ++ (solid) and HERWIG6 (dashed). Left/right panel: without/with acceptance cuts.

cuts (except in the case of figure 11, see below) we have required the top to have transverse momentum larger than $20 \mathrm{GeV}$, and the absolute value of its rapidity to be smaller than 2.5.

The distribution of the top-leading jet relative azimuth (figure 10) shows an increase in same-side emission, while the angular separation (measured in the rest frame of the top) between the charged lepton in leptonic top decays and the hardest non- $b$ jet (figure 11) tends to be slightly larger. In the right panel of figure 11, we have imposed the same acceptance cuts as in ref. [15]; namely, $p_{T}(b)>20 \mathrm{GeV},|\eta(b)|<2, p_{T}(l)>10 \mathrm{GeV}$, $|\eta(l)|<2.5, p_{T}(\nu)>20 \mathrm{GeV}, p_{T}(j)>20 \mathrm{GeV}$, and $|\eta(j)|<2.5$.

In accordance with the general tendency for more radiation in Herwig ++ , the overall jet activity (figure 12, left panel) is somewhat higher than in HERWIG6. The relative azimuthal distributions of the leading and next-to-leading jets (figure 12, right panel) are similar apart from an unexpected feature in the Herwig ++ distribution at low values. The corresponding jets have different rapidities but are aligned in azimuth. This appears to 

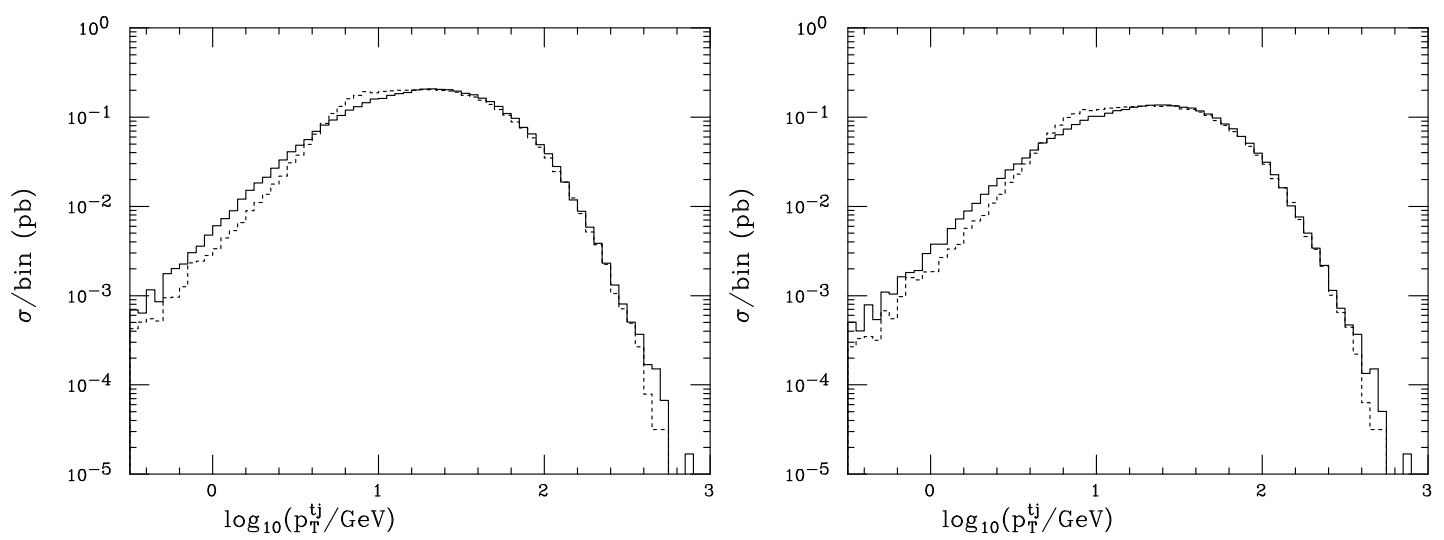

Figure 9. MC@NLO results on single top production: $\log _{10}$ of the transverse momentum distribution of the top-hardest jet pair, with Herwig ++ (solid) and HERWIG6 (dashed). Left/right panel: without/with acceptance cuts.
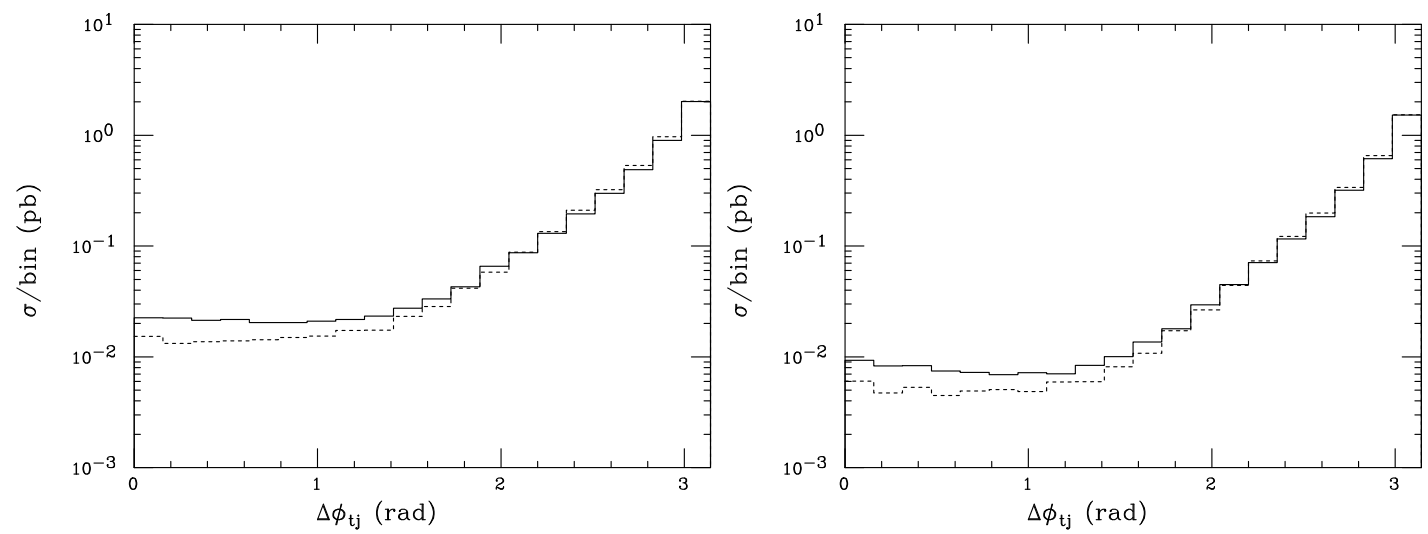

Figure 10. MC@NLO results on single top production: relative azimuth distribution between the top and the hardest jet, with Herwig ++ (solid) and HERWIG6 (dashed). Left/right panel: without/with acceptance cuts.

be a feature of the non-perturbative splitting of high-mass clusters connected to the beam remnant in Herwig ++. In order to show that this is the case, we overlay to the histograms in the right panel of figure 12 the corresponding results obtained by reconstructing jets at the parton level, i.e. before the non-perturbative gluon splitting (open boxes: Herwig++; open circles: HERWIG6). As can be seen from the figure, while the two HERWIG6 results are close to each other in the whole $\Delta \phi_{j j}$ range, the difference between the two Herwig ++ results at $\Delta \phi_{j j} \simeq 0$ is striking. It is therefore clear that the peak displayed there by the hadron-level results of Herwig++ is not related to the matching as performed by MC@NLO.

In other respects the treatment of non-perturbative effects looks more physical in Herwig ++ . Figures 13 and 14 show the transverse momentum and rapidity distributions of $b$-hadrons not from top decay, which come mainly from parton showering of initial-state $b$ quarks. In HERWIG6 these distributions have pathologies at low $p_{T}$ and high rapidity, 

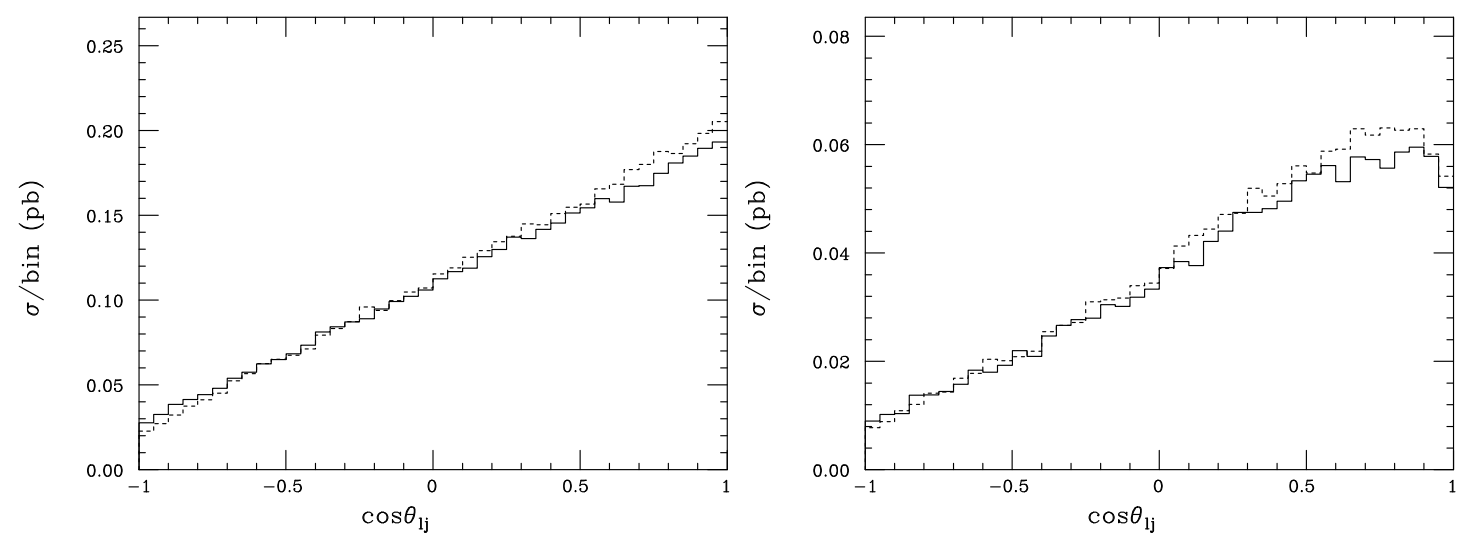

Figure 11. MC@NLO results on single top production: angle (in the top rest frame) between lepton from top decay and hardest non- $b$ jet with Herwig ++ (solid) and HERWIG6 (dashed). Left/right panel: without/with acceptance cuts.
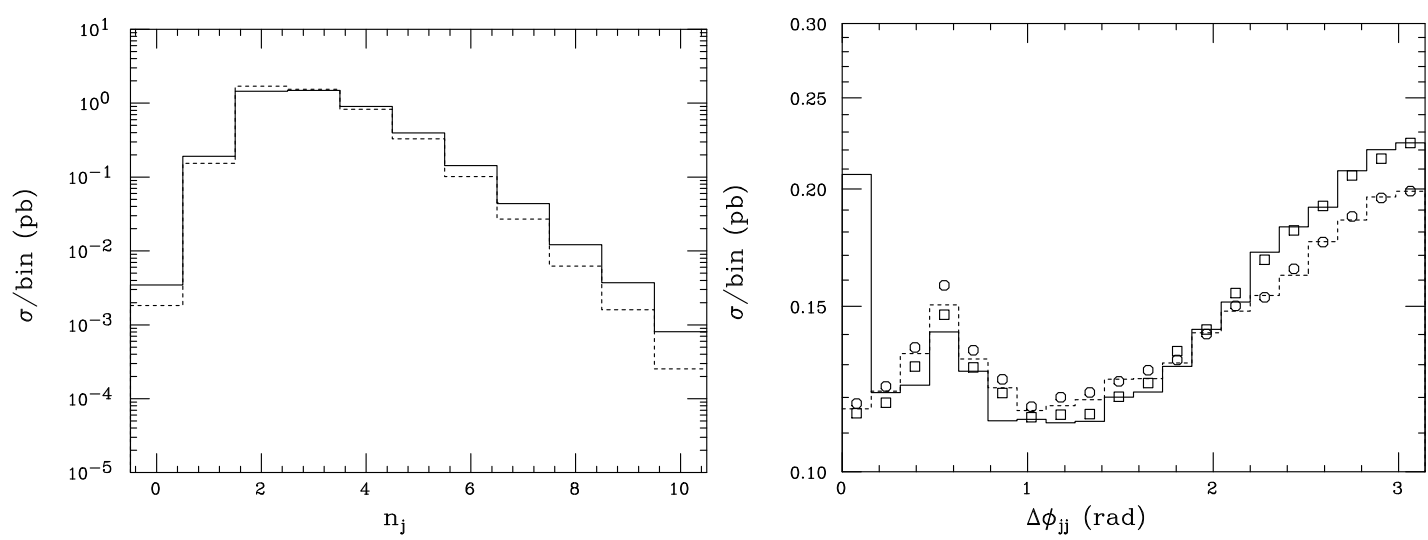

Figure 12. MC@NLO results on single top production: number of jets (left panel) and azimuthal separation between the two hardest jets (right panel), with Herwig ++ (solid) and HERWIG6 (dashed). Parton-level results for the azimuthal separation (open symbols) are presented as well: see the text for details.

arising from its simplified treatment of heavy quark showering and the model of nonperturbative $g \rightarrow b \bar{b}$ splitting used in matching the shower to the beam hadron. The latter gives rise to a deficit at $p_{T}<m_{b}$ and peaks around $|y| \sim 5$, which are less prominent in the model used in Herwig ++ , which has a smoother transition to the non-perturbative regime. As shown in the right-hand panels of figures 13 and 14, a cut on $y$ or $p_{T}$ removes most of the model dependence and yields much closer agreement between results from the two generators. In particular, in the kinematic region in which the $b$-hadrons are observable, the differences between Herwig ++ and HERWIG6 are small. This also implies that, owing to the fact that the inclusive $b$-hadron cross section predicted by the two PSMCs is identical (up to events with multiple $b$-hadrons, arising from $g \rightarrow b \bar{b}$ branchings in the shower), the impact of a veto cut will be similar in Herwig ++ and in HERWIG6. 

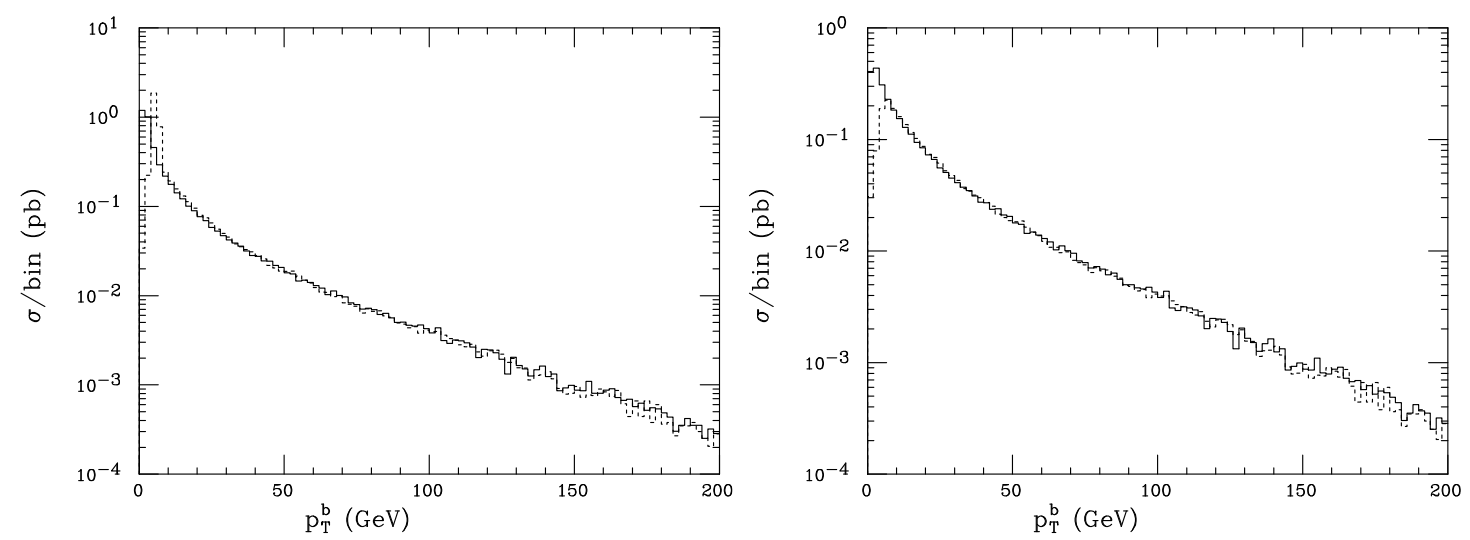

Figure 13. MC@NLO results on single top production: transverse momentum distributions of b-hadrons not from top decay with Herwig ++ (solid) and HERWIG6 (dashed). Left/right panels: without/with a cut on rapidity $|y|<3$.
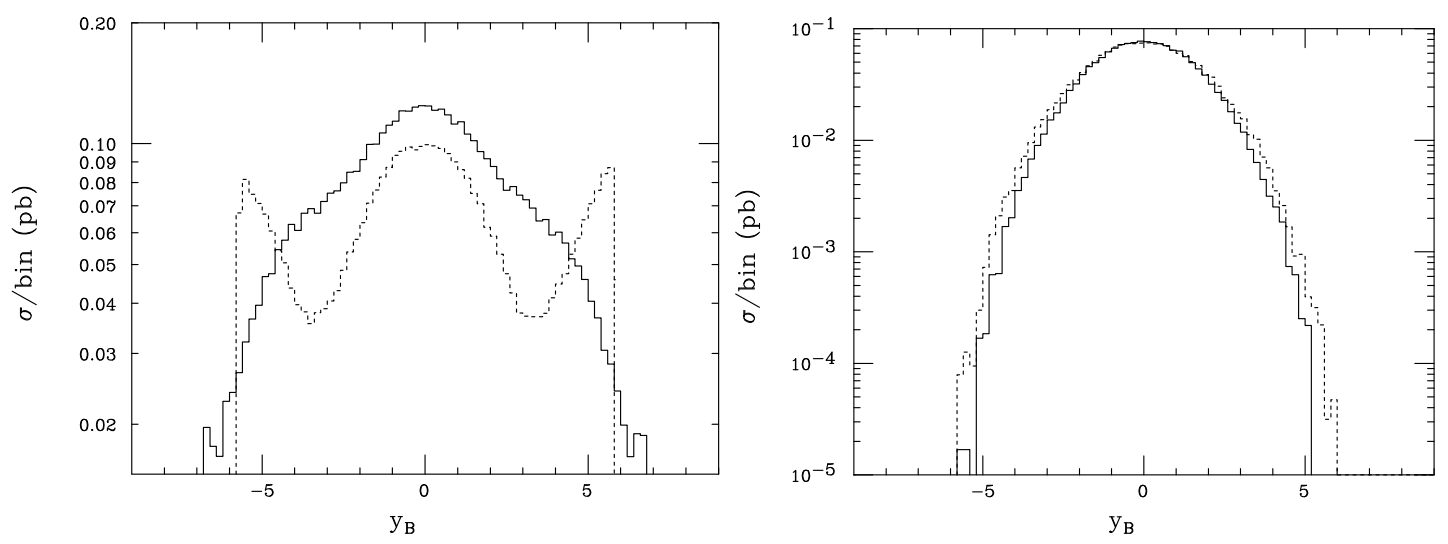

Figure 14. MC@NLO results on single top production: rapidity distributions of $b$-hadrons not from top decay with Herwig ++ (solid) and HERWIG6 (dashed). Left/right panels: without/with a cut on transverse momentum $p_{T}>10 \mathrm{GeV}$.

We conclude this section by mentioning that we have also considered single-top production in association with a $W$ boson, and compared Herwig++ with HERWIG6 results for both the DR and DS definitions of the $W t$ cross section (see ref. [24]). We did not attempt a full phenomenological study of this process, but limited ourselves to considering the impact of a $p_{T}$-veto imposed on the second-hardest $b$-hadron, which as discussed in ref. [24] is a rather effective way of reducing the $W t-t \bar{t}$ interference. We have found that Herwig ++ follows the same pattern as HERWIG6. This is reassuring, since it implies that the definition of $W t$ production as a separate process at the NLO is independent of the PSMC used in the simulations. 


\section{Conclusions}

In this paper we have presented the calculations necessary to match the Parton Shower Monte Carlo Herwig++ with any NLO QCD computation in the context of the MC@NLO formalism. The matching has then been achieved in practice for all processes which were already interfaced to Fortran HERWIG. A few selected MC@NLO/Herwig++ results obtained in this way have also been shown here, and compared to those obtained with MC@NLO/HERWIG.

From the technical point of view, the calculations performed here are of a complexity comparable with those reported in refs. [1, 11, 14], which were relevant to Fortran HERWIG. The corresponding computer programmes also behave in a fairly similar way. As expected, in those phase-space regions dominated by hard emissions, the results of MC@NLO/Herwig++ and MC@NLO/HERWIG6 coincide. On the other hand, differences (usually small) can be seen where multiple-parton emission plays a dominant role, with Herwig ++ typically giving a larger number of partons than HERWIG, but with smaller energies. In a very few cases larger discrepancies can be seen, and we have commented them in the text.

The results presented here provide all the ingredients needed for matching a lowmultiplicity NLO calculation to Herwig++. They also give the necessary and sufficient information for the matching of large-multiplicity processes, which we believe is best carried out in the context of a fully automated approach to NLO cross sections, and which we intend to pursue exploiting the work done in ref. [13].

\section{Acknowledgments}

We acknowledge the collaboration of Oluseyi Latunde-Dada and Peter Richardson in the early stages of this work, and thank them and other Herwig ++ authors for helpful discussions. B.R.W. is grateful to the CERN theory group for frequent hospitality. The work of P.T. is supported by the Swiss National Science Foundation.

\section{A Parton showering in Herwig ++}

As discussed in section 2.3, to evaluate the Monte Carlo subtraction terms (2.15) and (2.16) we need three kinds of information concerning the PSMC:

1. The shower variables, $z$ and $\tilde{q}^{2}$ in the case of Herwig ++ , and their expressions in terms of the variables used for the NLO calculation. In general, these expressions will be different for initial and final state showering, as indicated by eqs. (2.15) and (2.16) respectively.

2. The regions of phase space, if any, that are not covered by the parton showers at NLO (the so-called dead zones).

3. The splitting kernels used to generate the showers, $P_{a b}\left(z, \tilde{q}^{2}\right)$ in the case of Herwig ++ . 
The showering formalism adopted in Herwig ++ is that described in ref. [19]. We recall the relevant features here and derive the necessary formulae relating the shower variables to the invariants defined in earlier MC@NLO publications: see for example table 4 of ref. [14].

In Herwig ++ , as in all parton shower event generators, one starts with a (usually $2 \rightarrow 2$ ) hard subprocess and develops the external coloured legs into jets according to the showering formalism. The jets are then combined according to some kinematic reconstruction method that conserves 4-momentum, preserves the internal structure of the jets and in some sense maintains the configuration of the hard subprocess. This is not an unambiguous procedure, and Herwig++ includes options for different methods. We assume the one corresponding to the parameter values ReconstructionOption=General and (for initial-state showering) InitialInitialBoostOption=LongTransBoost. These ensure that the jets, once formed, are treated in the same way as in Fortran HERWIG, so the formulae in earlier MC@NLO papers apply once the invariant properties of a jet have been expressed in terms of the new shower variables.

Initial-state partons are always treated as massless in Herwig++, whereas final states may involve partons with non-zero, possibly unequal, masses. We treat the more complicated case of final-state showering first. Then in many (but not all) respects, the initialstate case simply corresponds to the massless limit of the same formalism.

\section{A.1 Final-state emission}

In showering from final-state partons, Herwig++ with MC@NLO runs in a reconstruction mode that preserves the 4 -momentum of the hard subprocess. This is achieved by aligning the shower axes with the directions of their parent partons in the subprocess rest frame and then boosting the showers along their axes by appropriate amounts. In order to be definite, we consider the case of the $2 \rightarrow 2$ Born subprocess

$$
a_{+}\left(\bar{p}_{1}\right)+a_{-}\left(\bar{p}_{2}\right) \longrightarrow a_{f_{1}}\left(\bar{k}_{1}\right)+a_{f_{2}}\left(\bar{k}_{2}\right)
$$

and the associated real-emission process

$$
a_{+}\left(p_{1}\right)+a_{-}\left(p_{2}\right) \longrightarrow a_{f_{1}}\left(k_{1}\right)+a_{f_{2}}\left(k_{2}\right)+a_{f_{3}}\left(k_{3}\right) .
$$

The masses of the outgoing partons $a_{f_{1}}$ and $a_{f_{2}}$ will be denoted by $m_{1}$ and $m_{2}$, while the initial-state partons $a_{ \pm}$and the emitted parton $a_{f_{3}}$ are always treated as massless. The $2 \rightarrow 2$ kinematics are defined by barred invariants:

$$
\begin{aligned}
& \bar{s}=2 \bar{p}_{1} \cdot \bar{p}_{2}=2 \bar{k}_{1} \cdot \bar{k}_{2}+m_{1}^{2}+m_{2}^{2}, \\
& \bar{t}=-2 \bar{p}_{1} \cdot \bar{k}_{1}=-2 \bar{p}_{2} \cdot \bar{k}_{2}-m_{1}^{2}+m_{2}^{2}, \\
& \bar{u}=-2 \bar{p}_{1} \cdot \bar{k}_{2}=-2 \bar{p}_{2} \cdot \bar{k}_{1}+m_{1}^{2}-m_{2}^{2}, \\
& 0=\bar{s}+\bar{t}+\bar{u} .
\end{aligned}
$$

The definitions of the $2 \rightarrow 3$ invariants used here are as in table 4 of ref. [14]. The relationships between the $2 \rightarrow 3$ and and $2 \rightarrow 2$ invariants implied by the Herwig++ kinematic reconstruction method are complicated but, as mentioned above, they are the same as in HERWIG6, so we also refer the reader to ref. [14] for them. 
The properties of an emission from a particular external line, say $a_{f_{1}} \rightarrow a_{f_{1}}+a_{f_{3}}$, that are invariant under kinematic reconstruction are: (i) the invariant mass of the pair $\left(k_{1}+k_{3}\right)^{2}$, and (ii) the ' + ' momentum fraction of the emitted massless parton,

$$
\zeta_{1} \equiv \frac{n \cdot k_{3}}{n \cdot\left(k_{1}+k_{3}\right)},
$$

where $n$ is a light-like reference vector antiparallel to the boost axis (i.e. along $a_{f_{2}}$ in this case). In terms of the invariants defined in ref. [14], i.e. $s=2 p_{1} \cdot p_{2}, w_{1}=2 k_{1} \cdot k_{3}$ and $w_{2}=2 k_{2} \cdot k_{3}$, we have

$$
\left(k_{1}+k_{3}\right)^{2}=w_{1}+m_{1}^{2}
$$

and one can prove (see ref. [14]) that the definition of $\zeta_{1}$ corresponds to

$$
\zeta_{1}=\frac{\left(2 s-\left(s-w_{1}\right) \epsilon_{2}\right) w_{2}+\left(s-w_{1}\right)\left[\left(w_{1}+w_{2}\right) \beta_{2}-\epsilon_{2} w_{1}\right]}{\left(s-w_{1}\right) \beta_{2}\left[2 s+\left(s-w_{1}\right)\left(\beta_{2}-\epsilon_{2}\right)\right]},
$$

with

$$
\begin{aligned}
& \epsilon_{2}=1-\frac{m_{1}^{2}-m_{2}^{2}}{s-w_{1}}, \\
& \beta_{2}=\sqrt{\epsilon_{2}^{2}-\frac{4 s m_{2}^{2}}{\left(s-w_{1}\right)^{2}}} .
\end{aligned}
$$

For showering by a final-state parton of mass $m_{1}$, the shower variables of Herwig ++ are

$$
\begin{aligned}
z & =\frac{n \cdot k_{1}}{n \cdot\left(k_{1}+k_{3}\right)}=1-\zeta_{1}, \\
\tilde{q}^{2} & =\frac{k_{T}^{2}}{z^{2}(1-z)^{2}}+\frac{m_{1}^{2}}{z^{2}},
\end{aligned}
$$

where $k_{T}$ is the transverse momentum of the emission, which is related to the invariant $w_{1}$ via eq. (A.5):

$$
k_{T}^{2}=(1-z)\left[z w_{1}-(1-z) m_{1}^{2}\right]
$$

so that

$$
\tilde{q}^{2}=\frac{w_{1}}{z(1-z)}=\frac{w_{1}}{\zeta_{1}\left(1-\zeta_{1}\right)} .
$$

In the case of emission from final-state parton $a_{f_{2}}$, one should simply replace $m_{1} \leftrightarrow m_{2}$ and $w_{1} \leftrightarrow w_{2}$.

The upper limit on the variable $\tilde{q}^{2}$, which sets the initial scale for the shower, is related to the colour connection structure of the $2 \rightarrow 2$ hard subprocess. If the final-state partons $a_{f_{1}}$ and $a_{f_{2}}$ are colour connected, as in $s$-channel single top production, the scale is set by the c.m. energy squared $\bar{s}=2 \bar{p}_{1} \cdot \bar{p}_{2}$. Since the hard subprocess 4-momentum is preserved in final-state emission, in this case we have $\bar{s}=s$. Then, as discussed in ref. [19], in order to yield the correct distribution of soft gluon radiation the regions filled by emissions from partons $a_{f_{1}}$ and $a_{f_{2}}$ should extend up to $\tilde{q}_{f_{1}}^{2}$ and $\tilde{q}_{f_{2}}^{2}$, respectively, where

$$
\left(\tilde{q}_{f_{1}}^{2}-m_{1}^{2}\right)\left(\tilde{q}_{f_{2}}^{2}-m_{2}^{2}\right)=\frac{1}{4}\left(s-m_{1}^{2}-m_{2}^{2}+\lambda\right)^{2} \quad \text { (final-final colour connection) }
$$


with

$$
\lambda=\lambda\left(\sqrt{s}, m_{1}, m_{2}\right) \equiv \sqrt{\left(s+m_{1}^{2}-m_{2}^{2}\right)^{2}-4 s m_{1}^{2}}=\sqrt{\left(s-m_{1}^{2}+m_{2}^{2}\right)^{2}-4 s m_{2}^{2}} .
$$

The default choice, which is adopted in MC@NLO, is to take

$$
\tilde{q}_{f_{1}}^{2}=\frac{1}{2}\left(s+m_{1}^{2}-m_{2}^{2}+\lambda\right), \quad \tilde{q}_{f_{2}}^{2}=\frac{1}{2}\left(s-m_{1}^{2}+m_{2}^{2}+\lambda\right) .
$$

If a given value of the NLO invariants corresponds to $\tilde{q}^{2}>\tilde{q}_{f_{\alpha}}^{2}$ for emission from parton $a_{f_{\alpha}}(\alpha=1$ or 2$)$, then that value lies in the dead zone for emission from that parton, and the corresponding MC subtraction term (2.16) vanishes there.

The splitting kernels used in Herwig++ include mass corrections appropriate to the quasi-collinear limit, as derived in ref. [20]. In terms of the above shower variables, for emission from parton $a_{f_{\alpha}}$, this limit corresponds to $m_{\alpha}, \tilde{q} \rightarrow 0$ with $m_{\alpha} / \tilde{q}$ finite. For the splitting $q \rightarrow q g$, we then have

$$
P_{q q}\left(z, \tilde{q}^{2}\right)=\frac{C_{F}}{1-z}\left[1+z^{2}-\frac{2 m_{q}^{2}}{z \tilde{q}^{2}}\right] .
$$

Recalling from eq. (A.8) that the collinear limit $k_{T} \rightarrow 0$ corresponds to $m_{q} / \tilde{q} \rightarrow z$, we see that emission extends down to zero angle, so that (unlike in HERWIG6) there is no empty "dead cone" around the collinear direction, although emission is suppressed in this region.

In the case of $g \rightarrow g g$ there are of course no mass corrections. For completeness, we note also the quasi-collinear kernel for $g \rightarrow q \bar{q}$,

$$
P_{q g}\left(z, \tilde{q}^{2}\right)=T_{R}\left[1-2 z(1-z)+\frac{2 m_{q}^{2}}{z(1-z) \tilde{q}^{2}}\right],
$$

although the mass correction for this splitting does not enter into any of the MC@NLO calculations considered here.

If the colour structure of the hard subprocess is such that an emitting final-state parton, say $a_{f_{1}}$, is colour connected to an initial-state parton, say $a_{+}$, the upper limit for its shower and that of $a_{+}$is set by the corresponding momentum transfer in the hard subprocess, given in this case by the variable $\bar{t}$, for which the expression in terms of $2 \rightarrow 3$ invariants may be found in ref. [14]. Again as discussed in ref. [19], in order to yield the correct distribution of soft gluon radiation the regions filled by emissions from partons $a_{+}$ and $a_{f_{1}}$ should extend up to $\tilde{q}_{+}^{2}$ and $\tilde{q}_{f_{1}}^{2}$, respectively, where now

$$
\tilde{q}_{+}^{2}\left(\tilde{q}_{f_{1}}^{2}-m_{1}^{2}\right)=\left(2 \bar{p}_{1} \cdot \bar{k}_{1}\right)^{2}=|\bar{t}|^{2} \quad \text { (initial-final colour connection). }
$$

In MC@NLO we use the default choice $\tilde{q}_{+}^{2}=|\bar{t}|, \tilde{q}_{f_{1}}^{2}=|\bar{t}|+m_{1}^{2}$.

If the colour connection of $a_{f_{1}}$ is instead to parton $a_{-}$, then $|\bar{t}|$ is replaced by $2 \bar{p}_{2} \cdot \bar{k}_{1}=$ $|\bar{u}|+m_{1}^{2}-m_{2}^{2}$. Similarly, if parton $a_{f_{2}}$ is connected to $a_{+}$, we have

$$
\tilde{q}_{+}^{2}\left(\tilde{q}_{f_{2}}^{2}-m_{2}^{2}\right)=\left(2 \bar{p}_{1} \cdot \bar{k}_{2}\right)^{2}=|\bar{u}|^{2},
$$

with the default choice $\tilde{q}_{+}^{2}=|\bar{u}|, \tilde{q}_{f_{2}}^{2}=|\bar{u}|+m_{2}^{2}$, while if $a_{f_{2}}$ is connected to $a_{-}$, then $|\bar{u}|$ is replaced by $2 \bar{p}_{2} \cdot \bar{k}_{2}=|\bar{t}|-m_{1}^{2}+m_{2}^{2}$.

We discuss in the next subsection how the shower variable $\tilde{q}^{2}$ is defined for initial-state emission. 


\section{A.2 Initial-state emission}

In showering from initial-state partons, the 4-momentum of the hard subprocess is not preserved: it recoils longitudinally and transversely from the emissions in the showers. Herwig++ with MC@NLO runs in a reconstruction mode that preserves the invariant mass and rapidity of the hard subprocess. Considering again the $2 \rightarrow 2$ hard subprocess (A.1) and the corresponding real emission (A.2), suppose that the massless parton $a_{f_{3}}$ is emitted from $a_{+}$, also taken to be massless. The Herwig ++ variables for an initial-state shower are

$$
z=1-\frac{n \cdot k_{3}}{n \cdot p_{1}}, \quad \tilde{q}^{2}=\frac{k_{T}^{2}}{(1-z)^{2}}
$$

Since the two initial-state showers are (anti-)aligned and the other incoming massless parton $a_{-}$does not emit, we can take $n=p_{2}$. In terms of the invariants of [14], i.e. $s=2 p_{1} \cdot p_{2}$, $v_{1}=-2 p_{1} \cdot k_{3}$ and $v_{2}=-2 p_{2} \cdot k_{3}$, we then have

$$
z=1+\frac{v_{2}}{s}
$$

and $k_{T}^{2}=-(1-z) v_{1}$, so that

$$
\tilde{q}^{2}=\frac{v_{1} s}{v_{2}} .
$$

For emission from incoming parton $a_{-}$, interchange $v_{1} \leftrightarrow v_{2}$.

For subprocesses where the initial-state partons $a_{+}$and $a_{-}$are colour connected, the scales for their showers are set by the hard subprocess invariant mass squared, $\bar{s}$. The showers from partons $a_{+}$and $a_{-}$should extend up to $\tilde{q}_{+}^{2}$ and $\tilde{q}_{-}^{2}$ such that

$$
\tilde{q}_{+}^{2} \tilde{q}_{-}^{2}=\bar{s}^{2} \quad \text { (initial-initial colour connection) }
$$

and we adopt the default values $\tilde{q}_{+}^{2}=\tilde{q}_{-}^{2}=\bar{s}$. This looks similar to the case of final-final colour connection for massless partons, eq. (A.11). Note however that in the present case we have $\bar{s} \neq s$ due to recoil effects; in fact

$$
\bar{s}=s+v_{1}+v_{2} .
$$

If on the other hand the colour connection is from $a_{+}$to final-state parton $a_{f_{1}}$, then we follow the prescription (A.16) instead of (A.21), and the upper limit on $\tilde{q}_{+}^{2}$ is given by $\tilde{q}_{+}^{2}=|\bar{t}|$. If the colour connection is instead to parton $a_{f_{2}}$, then the limit is $|\bar{u}|$. The limits for initial-final connections of parton $a_{-}$follow analogously.

Table 2 summarizes the limits on $\tilde{q}^{2}$ for all the possible colour connections. If the emitted parton $a_{f_{3}}$ is a light quark instead of a gluon, as can happen in initial-state showering, for example in $g q \rightarrow Z^{0} q$, the same limits apply, even though the soft gluon radiation pattern is not relevant. This is because Herwig++ evolves the shower downwards from the limiting scale and does not determine in advance whether a quark or a gluon will be emitted in the first splitting. 


\begin{tabular}{|c|c|l|}
\hline Parton & Col. con. & Limit \\
\hline \hline$a_{+}$ & $a_{-}$ & $\bar{s}$ \\
$a_{+}$ & $a_{f_{1}}$ & $|\bar{t}|$ \\
$a_{+}$ & $a_{f_{2}}$ & $|\bar{u}|$ \\
\hline$a_{-}$ & $a_{+}$ & $\bar{s}$ \\
$a_{-}$ & $a_{f_{1}}$ & $|\bar{u}|+m_{1}^{2}-m_{2}^{2}$ \\
$a_{-}$ & $a_{f_{2}}$ & $|\bar{t}|-m_{1}^{2}+m_{2}^{2}$ \\
\hline$a_{f_{1}}$ & $a_{+}$ & $|\bar{t}|+m_{1}^{2}$ \\
$a_{f_{1}}$ & $a_{-}$ & $|\bar{u}|+2 m_{1}^{2}-m_{2}^{2}$ \\
$a_{f_{1}}$ & $a_{f_{2}}$ & $\frac{1}{2}\left(s+m_{1}^{2}-m_{2}^{2}+\lambda\right)$ \\
\hline$a_{f_{2}}$ & $a_{+}$ & $|\bar{u}|+m_{2}^{2}$ \\
$a_{f_{2}}$ & $a_{-}$ & $|\bar{t}|-m_{1}^{2}+2 m_{2}^{2}$ \\
$a_{f_{2}}$ & $a_{f_{1}}$ & $\frac{1}{2}\left(s-m_{1}^{2}+m_{2}^{2}+\lambda\right)$ \\
\hline
\end{tabular}

Table 2. Limits on $\tilde{q}^{2}$ for showering partons with different colour connections. The kinematic invariants are defined in eqs. (A.3) and (A.12).

\section{B Construction of MC subtraction terms}

In this section we again consider the case of the real-emission process (A.2) and label the relevant $\mathcal{S}$ functions by

$$
m_{ \pm}=\left\{\left(f_{3},+\right),\left(f_{3},-\right)\right\}, \quad m_{1}=\left(f_{3}, f_{1}\right), \quad m_{2}=\left(f_{3}, f_{2}\right)
$$

which is more general than either eq. (2.9) or eq. (2.10), and in fact allows one to deal with any $2 \rightarrow 3$ real-emission process (possibly after relabeling of the partons in eq. (A.2)). The subtracted real-emission contribution to the NLO cross section read $[12,13]$

$$
\begin{aligned}
d \hat{\sigma}_{\mu \mid m_{ \pm}}^{(3)} & =\frac{1}{2}\left(\frac{1}{\xi}\right)_{c}\left[\left(\frac{1}{1-y_{ \pm}}\right)_{\delta}+\left(\frac{1}{1+y_{ \pm}}\right)_{\delta}\right]\left(\left(1-y_{ \pm}^{2}\right) \xi^{2} \mathcal{M}_{\mu}^{(3)}\right) \mathcal{S}_{\mu \mid m_{ \pm}} \\
& \times d \xi d y_{ \pm} d \varphi_{ \pm} d \tilde{\phi}_{2}^{\left(m_{ \pm}\right)} \\
d \hat{\sigma}_{\mu \mid m_{f_{\alpha}}}^{(3)} & =\left(\frac{1}{\xi}\right)_{c}\left(\frac{1}{1-y_{f_{\alpha}}}\right)_{\delta}\left(\left(1-y_{f_{\alpha}}\right) \xi^{2} \mathcal{M}_{\mu}^{(3)}\right) \mathcal{S}_{\mu \mid m_{f_{\alpha}}} d \xi d y_{f_{\alpha}} d \varphi_{f_{\alpha}} d \tilde{\phi}_{2}^{\left(m_{f_{\alpha}}\right)} .
\end{aligned}
$$

The variables used in these equations are always defined in the c.m. frame of the colliding partons. We have denoted by $\xi$ the energy of parton $a_{f_{3}}$, divided by $\sqrt{s} / 2$, with $s$ the c.m. energy squared. In eq. (B.2), $y_{ \pm}$denotes the cosine of the angle between partons $a_{f_{3}}$ and $a_{+}$, while in eq. (B.3) $y_{f_{\alpha}}$ denotes the cosine of the angle between partons $a_{f_{3}}$ and $a_{f_{\alpha}}$. The quantities $\varphi_{ \pm}$and $\varphi_{f_{\alpha}}$ are azimuthal angles, whose definitions are not relevant in what follows. The plus distributions in $\xi, y_{ \pm}$, and $y_{f_{\alpha}}$ that appear in eqs. (B.2) and (B.3) subtract the soft, initial-state collinear, and final-state collinear singularities respectively. 
By construction, the three-body phase space is

$$
\begin{aligned}
d \phi_{3} & =\xi d \xi d y_{ \pm} d \varphi_{ \pm} d \tilde{\phi}_{2}^{\left(m_{ \pm}\right)} \\
& =\xi d \xi d y_{f_{\alpha}} d \varphi_{f_{\alpha}} d \tilde{\phi}_{2}^{\left(m_{f_{\alpha}}\right)},
\end{aligned}
$$

where the measures $d \tilde{\phi}_{2}^{(m)}$ have the same dimensionality of the two-body phase space $d \phi_{2}$, and are proportional to it in the relevant soft and collinear limits. Using eqs. (B.4) and (B.5), we see that the unsubtracted real-emission cross sections (i.e., the quantities obtained from eqs. (B.2) and (B.3) by replacing the plus distributions with ordinary functions) are such that

$$
\begin{aligned}
d \sigma_{\mu \mid m_{ \pm}}^{(3)} & =\mathcal{M}_{\mu}^{(3)} \mathcal{S}_{\mu \mid m_{ \pm}} d \phi_{3} \\
d \sigma_{\mu \mid m_{f_{\alpha}}}^{(3)} & =\mathcal{M}_{\mu}^{(3)} \mathcal{S}_{\mu \mid m_{f_{\alpha}}} d \phi_{3} .
\end{aligned}
$$

Hence, one defines

$$
d \bar{\Sigma}_{\mu \mid m_{ \pm}}^{(3)}=\mathcal{L} d \sigma_{\mu \mid m_{ \pm}}^{(3)}, \quad d \bar{\Sigma}_{\mu \mid m_{f_{\alpha}}}^{(3)}=\mathcal{L} d \sigma_{\mu \mid m_{f_{\alpha}}}^{(3)},
$$

which is eq. (2.3).

The equations given above can now be used for the explicit construction of the MC subtraction terms $d \bar{\Sigma}_{\mu \mid m}^{(\mathrm{MC})}$ used in the MC@NLO generating functional, eq. (2.5). In particular, the idea is to express the short-distance cross sections of $\mathrm{MC}$ origin, eqs. (2.15) and (2.16), in the same form as the NLO ones, eqs. (B.2) and (B.3). Then, these cross sections are multiplied by the luminosity factors that appear in eqs. (2.12)-(2.14) to obtain the MC subtraction terms, by analogy with eq. (B.8).

The manipulations of the MC short-distance cross section are based on the following observations. Firstly, the variables $\xi$ and $y_{ \pm}$(or $\xi$ and $y_{f_{\alpha}}$ ), introduced in the FKS subtraction method for the integration of the NLO cross sections, are in one-to-one correspondence with the Herwig ++ shower variables $z_{ \pm}$and $\tilde{q}_{ \pm}^{2}$ (or $z_{f_{\alpha}}$ and $\tilde{q}_{f_{\alpha}}^{2}$ ); hence, the two pairs can be related by a change of variables. Secondly, the Born-level cross sections that appear in eqs. (2.15) and (2.16) can be written as [25]

$$
d \bar{\sigma}_{\mu^{\prime}}^{(L, l)}=\overline{\mathcal{M}}_{\mu^{\prime}}^{(b ; L, l)} d \phi_{2},
$$

with

$$
\overline{\mathcal{M}}_{\mu^{\prime}}^{(b ; L, l)}=\frac{1}{\mathcal{N}_{L}} \frac{D_{f(L, l)}}{\sum_{f^{\prime}} D_{f^{\prime}}} \mathcal{M}_{\mu^{\prime}}^{(b)}, \quad \lim _{N_{c} \rightarrow \infty} \mathcal{M}_{\mu^{\prime}}^{(b)}=\sum_{f^{\prime}} D_{f^{\prime}}
$$

Here, $D_{f^{\prime}}$ is the leading- $N_{c}$ contribution to the Born matrix element squared, $\mathcal{M}_{\mu^{\prime}}^{(b)}$, for a given colour flow $f^{\prime}$, with $f(L, l)$ the colour flow identified by $L$ and $l$. The pre-factor $\mathcal{N}_{L}$ is equal to one or two in the case of a branching of a quark or a gluon line respectively (the latter choice is due to the fact that a gluon has two colour partners for a given colour flow, and one of them is chosen at random with probability equal to $1 / 2$ ). In eq. (B.9), $d \phi_{2}$ is the two-body phase-space, whose explicit parametrization depends on the leg that 
will eventually branch. It is clear, thus, that such a phase-space can easily be related to $d \tilde{\phi}_{2}^{\left(m_{ \pm}\right)}$or to $d \tilde{\phi}_{2}^{\left(m_{f_{\alpha}}\right)}$.

We start by dealing with eq. (2.15), which we re-write as follows:

$$
\begin{aligned}
& \left.d \hat{\sigma}_{\mu}^{( \pm, l)}\right|_{\mathrm{MC}}=\frac{1}{2 \xi}\left[\frac{1}{1-y_{ \pm}}+\frac{1}{1+y_{ \pm}}\right]\left(\left.\left(1-y_{ \pm}^{2}\right) \xi^{2} \frac{d \varsigma_{\mu}^{( \pm, l)}}{d \phi_{3}}\right|_{\mathrm{MC}}\right) \frac{d \phi_{3}}{\xi} \\
& \left.\frac{d \varsigma_{\mu}^{( \pm, l)}}{d \phi_{3}}\right|_{\mathrm{MC}}=\frac{\alpha_{S}}{(2 \pi)^{2}} \frac{P_{a^{\prime} b^{\prime}}\left(z_{ \pm}\right)}{\xi \tilde{q}_{ \pm}^{2}} \overline{\mathcal{M}}_{\mu^{\prime}}^{(b ; \pm, l)} \Theta_{\text {dead }}^{( \pm, l)} \frac{\partial\left(z_{ \pm}, \tilde{q}_{ \pm}^{2}\right)}{\partial\left(\xi, y_{ \pm}\right)} \frac{d \phi_{2}}{d \tilde{\phi}_{2}^{\left(m_{ \pm}\right)}} .
\end{aligned}
$$

In order to be able to express the measure that appears in eq. (2.15) in terms of the threebody phase space $d \phi_{3}$, we have inserted a trivial factor $d \varphi_{ \pm} /(2 \pi)$ on its r.h.s., and made use of eq. (B.4). We can now insert the luminosity factors. Equations (2.12) and (2.13) suggest we define

$$
\begin{aligned}
& d \mathcal{L}^{(+)}=\mathcal{L}^{(+)} d \bar{x}_{1 i} d \bar{x}_{2 i} \equiv \frac{1}{z_{+}} f_{a}^{\left(H_{1}\right)}\left(\bar{x}_{1 i} / z_{+}\right) f_{b}^{\left(H_{2}\right)}\left(\bar{x}_{2 i}\right) d \bar{x}_{1 i} d \bar{x}_{2 i}, \\
& d \mathcal{L}^{(-)}=\mathcal{L}^{(-)} d \bar{x}_{1 i} d \bar{x}_{2 i} \equiv \frac{1}{z_{-}} f_{a}^{\left(H_{1}\right)}\left(\bar{x}_{1 i}\right) f_{b}^{\left(H_{2}\right)}\left(\bar{x}_{2 i} / z_{-}\right) d \bar{x}_{1 i} d \bar{x}_{2 i} .
\end{aligned}
$$

As discussed in section 2.3, when matching a PSMC with an NLO computation, variables $\bar{x}_{1 i}$ and $\bar{x}_{2 i}$ can be expressed in terms of their analogues used in the NLO computation (which we have denoted by $x_{1}$ and $x_{2}$ in eq. (2.4)). Hence we can write

$$
d \mathcal{L}^{( \pm)}=\mathcal{L}^{( \pm)} \frac{\partial\left(\bar{x}_{1 i}, \bar{x}_{2 i}\right)}{\partial\left(x_{1}, x_{2}\right)} d x_{1} d x_{2} .
$$

Putting everything together, we are led to define

$$
\frac{d \bar{\Sigma}_{\mu \mid m_{ \pm}}^{(\mathrm{MC})}}{d \phi_{3}}=\frac{\partial\left(\bar{x}_{1 i}, \bar{x}_{2 i}\right)}{\partial\left(x_{1}, x_{2}\right)} \sum_{l}\left(\left.\mathcal{L}^{(+)} \frac{d \varsigma_{\mu}^{(+, l)}}{d \phi_{3}}\right|_{\mathrm{MC}}+\left.\mathcal{L}^{(-)} \frac{d \varsigma_{\mu}^{(-, l)}}{d \phi_{3}}\right|_{\mathrm{MC}}\right) .
$$

We explicitly point out that, as the notation suggests, the variables $\bar{x}_{1 i}$ and $\bar{x}_{2 i}$ have the same functional form w.r.t. $x_{1}$ and $x_{2}$ regardless of whether the branching parton is $a_{+}$or $a_{-}$, and therefore one is able to factor out the jacobian factor in eq. (B.16). We also stress that the damping factor $\left(1-y_{ \pm}^{2}\right) \xi^{2}$ that appears in eq. (B.11) is cancelled by an identical factor in the denominator of that equation. The reason for writing eq. (B.11) in that way is to make an explicit connection with its real-emission counterpart, eq. (B.2). In fact, in numerical codes it turns out to be convenient to define as core functions the real-emission matrix elements, or the MC subtraction terms, times the damping factor, for the simple reason that these quantities are finite in the soft and collinear limits.

The treatment of final-state branchings is completely analogous to that discussed above. We have

$$
\begin{aligned}
\left.d \hat{\sigma}_{\mu}^{\left(f_{\alpha}, l\right)}\right|_{\mathrm{MC}} & =\frac{1}{\xi} \frac{1}{1-y_{f_{\alpha}}}\left(\left.\left(1-y_{f_{\alpha}}\right) \xi^{2} \frac{d \varsigma_{\mu}^{\left(f_{\alpha}, l\right)}}{d \phi_{3}}\right|_{\mathrm{MC}}\right) \frac{d \phi_{3}}{\xi} \\
\left.\frac{d \varsigma_{\mu}^{\left(f_{\alpha}, l\right)}}{d \phi_{3}}\right|_{\mathrm{MC}} & =\frac{\alpha_{S}}{(2 \pi)^{2}} \frac{P_{a^{\prime} b^{\prime}}\left(z_{f_{\alpha}}, \tilde{q}_{f_{\alpha}}^{2}\right)}{\xi \tilde{q}_{f_{\alpha}}^{2}} \overline{\mathcal{M}}_{\mu^{\prime}}^{\left(b ; f_{\alpha}, l\right)} \Theta_{\text {dead }}^{\left(f_{\alpha}, l\right)} \frac{\partial\left(z_{f_{\alpha}}, \tilde{q}_{f_{\alpha}}^{2}\right)}{\partial\left(\xi, y_{f_{\alpha}}\right)} \frac{d \phi_{2}}{d \tilde{\phi}_{2}^{\left(m_{f_{\alpha}}\right)}} .
\end{aligned}
$$


Furthermore, from eq. (2.14) we have

$$
d \mathcal{L}^{\left(f_{\alpha}\right)}=\mathcal{L}^{\left(f_{\alpha}\right)} d \bar{x}_{1 f} d \bar{x}_{2 f}, \equiv f_{a}^{\left(H_{1}\right)}\left(\bar{x}_{1 f}\right) f_{b}^{\left(H_{2}\right)}\left(\bar{x}_{2 f}\right) d \bar{x}_{1 f} d \bar{x}_{2 f} .
$$

As discussed in ref. [14], in the case of final-state branchings we have $\bar{x}_{1 f}=x_{1}$ and $\bar{x}_{2 f}=x_{2}$. Therefore

$$
\frac{d \bar{\Sigma}_{\mu \mid m_{f_{\alpha}}}^{(\mathrm{MC})}}{d \phi_{3}}=\left.\sum_{l} \mathcal{L}^{\left(f_{\alpha}\right)} \frac{d \varsigma_{\mu}^{\left(f_{\alpha}, l\right)}}{d \phi_{3}}\right|_{\mathrm{MC}} .
$$

Open Access. This article is distributed under the terms of the Creative Commons Attribution Noncommercial License which permits any noncommercial use, distribution, and reproduction in any medium, provided the original author(s) and source are credited.

\section{References}

[1] S. Frixione and B.R. Webber, Matching NLO QCD computations and parton shower simulations, JHEP 06 (2002) 029 [hep-ph/0204244] [SPIRES].

[2] G. Marchesini et al., HERWIG: a Monte Carlo event generator for simulating hadron emission reactions with interfering gluons. Version 5.1 - April 1991, Comput. Phys. Commun. 67 (1992) 465 [SPIRES].

[3] G. Corcella et al., HERWIG 6.5: an event generator for Hadron Emission Reactions With Interfering Gluons (including supersymmetric processes), JHEP 01 (2001) 010 [hep-ph/0011363] [SPIRES].

[4] G. Corcella et al., HERWIG 6.5 release note, hep-ph/0210213 [SPIRES].

[5] P. Torrielli and S. Frixione, Matching NLO QCD computations with PYTHIA using MC@NLO, JHEP 04 (2010) 110 [arXiv: 1002.4293] [SPIRES].

[6] M. Bahr et al., HERWIG++ physics and manual, Eur. Phys. J. C 58 (2008) 639 [arXiv:0803.0883] [SPIRES].

[7] M. Bahr et al., HERWIG ++2.3 release note, arXiv:0812. 0529 [SPIRES].

[8] O. Latunde-Dada, HERWIG Monte Carlo at Next-to-Leading Order for $e^{+} e^{-}$annihilation and lepton pair production, JHEP 11 (2007) 040 [arXiv:0708.4390] [SPIRES].

[9] A. Papaefstathiou and O. Latunde-Dada, NLO production of $W^{\prime}$ bosons at hadron colliders using the MC@NLO and POWHEG methods, JHEP 07 (2009) 044 [arXiv:0901.3685] [SPIRES].

[10] O. Latunde-Dada, MC@NLO for the hadronic decay of Higgs bosons in associated production with vector bosons, JHEP 05 (2009) 112 [arXiv: 0903.4135] [SPIRES].

[11] S. Frixione, P. Nason and B.R. Webber, Matching NLO QCD and parton showers in heavy flavour production, JHEP 08 (2003) 007 [hep-ph/0305252] [SPIRES].

[12] S. Frixione, Z. Kunszt and A. Signer, Three jet cross-sections to next-to-leading order, Nucl. Phys. B 467 (1996) 399 [hep-ph/9512328] [SPIRES].

[13] R. Frederix, S. Frixione, F. Maltoni and T. Stelzer, Automation of next-to-leading order computations in QCD: the FKS subtraction, JHEP 10 (2009) 003 [arXiv:0908.4272] [SPIRES]. 
[14] S. Frixione, E. Laenen, P. Motylinski and B.R. Webber, Single-top production in MC@NLO, JHEP 03 (2006) 092 [hep-ph/0512250] [SPIRES].

[15] S. Frixione, E. Laenen, P. Motylinski and B.R. Webber, Angular correlations of lepton pairs from vector boson and top quark decays in Monte Carlo simulations, JHEP 04 (2007) 081 [hep-ph/0702198] [SPIRES].

[16] P.M. Nadolsky et al., Implications of CTEQ global analysis for collider observables, Phys. Rev. D 78 (2008) 013004 [arXiv:0802.0007] [SPIRES].

[17] C. Anastasiou, G. Dissertori, F. Stockli and B.R. Webber, QCD radiation effects on the

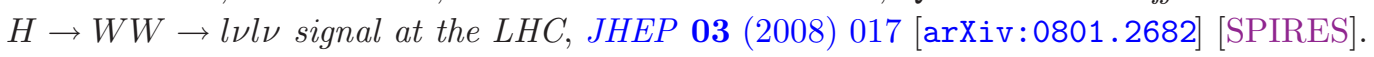

[18] C. Anastasiou, G. Dissertori, M. Grazzini, F. Stockli and B.R. Webber, Perturbative QCD effects and the search for $a H \rightarrow W W \rightarrow l \nu l \nu$ signal at the Tevatron, JHEP 08 (2009) 099 [arXiv:0905.3529] [SPIRES].

[19] S. Gieseke, P. Stephens and B. Webber, New formalism for QCD parton showers, JHEP 12 (2003) 045 [hep-ph/0310083] [SPIRES].

[20] S. Catani, S. Dittmaier and Z. Trócsányi, One-loop singular behaviour of QCD and SUSY QCD amplitudes with massive partons, Phys. Lett. B 500 (2001) 149 [hep-ph/0011222] [SPIRES].

[21] K. Hamilton and P. Richardson, A simulation of QCD radiation in top quark decays, JHEP 02 (2007) 069 [hep-ph/0612236] [SPIRES].

[22] M. Cacciari and G.P. Salam, Dispelling the $N^{3}$ myth for the $k_{t}$ jet-finder, Phys. Lett. B 641 (2006) 57 [hep-ph/0512210] [SPIRES].

[23] M. Cacciari, G.P. Salam and G. Soyez, FastJet, http://fastjet.fr/.

[24] S. Frixione, E. Laenen, P. Motylinski, B.R. Webber and C.D. White, Single-top hadroproduction in association with a $W$ boson, JHEP 07 (2008) 029 [arXiv:0805.3067] [SPIRES].

[25] K. Odagiri, Color connection structure of supersymmetric $Q C D(2 \rightarrow 2)$ processes, JHEP 10 (1998) 006 [hep-ph/9806531] [SPIRES]. 